\title{
Cross-trait analysis of Alzheimer's disease and gastrointestinal tract disorders identifies shared loci highlighting immune-related and statin pathways
}

Emmanuel Adewuyi ( $\nabla$ e.adewuyi@ecu.edu.au )

Edith Cowan University https://orcid.org/0000-0002-4533-0340

Eleanor O'Brien

Edith Cowan University

Dale Nyholt

Queensland University of Technology https://orcid.org/0000-0001-7159-3040

Tenielle Porter

Edith Cowan University

Simon Laws

Edith Cowan University https://orcid.org/0000-0002-4355-7082

Article

Keywords: Alzheimer's disease, gastrointestinal tract disorders, cross-trait analysis, genome-wide association studies

Posted Date: August 6th, 2021

DOl: https://doi.org/10.21203/rs.3.rs-757229/v1

License: (c) (1) This work is licensed under a Creative Commons Attribution 4.0 International License. Read Full License 
1 Cross-trait analysis of Alzheimer's disease and gastrointestinal tract disorders

2 identifies shared loci highlighting immune-related and statin pathways

3

4 Emmanuel O Adewuyi ${ }^{1,2^{*}}$, Eleanor K. O’Brien ${ }^{1,2}$, Dale R Nyholt ${ }^{3}$, Tenielle Porter ${ }^{1,2,4}$, Simon M 5 Laws L $^{1,2,4^{*}}$

$6 \quad{ }^{1}$ Centre for Precision Health, Edith Cowan University, Joondalup 6027, Western Australia

$7 \quad{ }^{2}$ Collaborative Genomics and Translation Group, School of Medical and Health Sciences, Edith

8 Cowan University, Joondalup 6027, Western Australia

$9{ }^{3}$ Centre for Genomics and Personalised Health, School of Biomedical Sciences, Faculty of

10 Health, Queensland University of Technology, Brisbane, Queensland, Australia

$11{ }^{4}$ Curtin Health Innovation Research Institute, Curtin University, Bentley, 6102, Western

12 Australia

*Corresponding authors:

Dr. Emmanuel Adewuyi, Centre for Precision Health, Edith Cowan University, 270 Joondalup Drive, Joondalup 6027, Western Australia; e.adewuyi@ecu.edu.au

Prof. Simon M Laws, Centre for Precision Health, Edith Cowan University, 270 Joondalup Drive, Joondalup 6027, Western Australia; +61 86304 5128; s.laws@ecu.edu.au; @Simon_M_Laws 
39

Several observational studies suggest a relationship between Alzheimer's disease (AD) and gastrointestinal tract (GIT) disorders; however, their underlying mechanisms remain unclear. Here, we analysed several genome-wide association studies (GWAS) summary statistics ( $N=$ $34,652-456,327)$ to assess AD and GIT disorders relationships. We found a significant genetic overlap and correlation between $A D$ and each of gastroesophageal reflux disease (GERD), peptic ulcer disease (PUD), medications for GERD or PUD (PGM), gastritis-duodenitis, irritable bowel syndrome and diverticulosis, but not inflammatory bowel disease. Our analysis suggests a partial causal association between $A D$ and gastritis-duodenitis, diverticulosis and medication for PUD. GWAS meta-analysis identified seven loci $\left(\mathrm{P}<5 \times 10^{-8}, P D E 4 B, C D 46\right.$, SEMA3F, HLA-DRA, MTSS2, PHB, and APOE) shared by AD and PGM, six of which are novel. These loci were replicated using GERD and PUD GWAS and reinforced in gene-based analyses. Lipid metabolism, autoimmune system, lipase inhibitors, PD-1 signalling, and statin pathways were significantly enriched for $A D$ and GIT disorders. These findings support shared genetic susceptibility in AD and GIT disorders. Lipase inhibitors and statins may provide novel therapeutic avenues for AD, GIT disorders, or their comorbidity. 
Alzheimer's disease (AD) is the most prevalent form of dementia, characterised by neurodegeneration and a progressive decline in cognitive ability beyond what would be expected from the normal ageing process ${ }^{1,2}$. The disorder ranks as a subject of significant global public health importance with consequences for wide-ranging social and economic adverse impacts on sufferers, their families, and the society at large ${ }^{1}$. By the year 2030 , over 82 million people-and about 152 million by 2050 -are projected to suffer from $A D^{1,2}$. The annual global economic costs of the disorder are currently estimated at nearly one trillion US dollars and predicted to reach two trillion US dollars in $2030^{1-4}$. While AD has no known curative treatments, and its pathogenesis is yet to be clearly understood, a comprehensive assessment of its shared genetics with diseases (comorbidities) can provide a deeper understanding of its underlying biological mechanisms and those of its comorbidities.

Several studies have reported a pattern of co-occurrence of dementia (and AD in particular) with certain gastrointestinal tract (GIT) disorders, microbiota or medications commonly used in the treatment of peptic ulcer disease (PUD) ${ }^{5-11}$. For example, an observational study reported more than twice the odds of dementia in individuals with gastritis (adjusted odds ratio (OR): $2.42, \mathrm{P}<0.001,95 \%$ confidence interval $(\mathrm{Cl}): 1.68-3.49)^{5}$. Another observational medications for the treatment of gastritis-duodenitis, gastroesophageal reflux disease (GERD) or PUD) and increased risk of incident dementia (hazard ratio [HR]: 1.44 [95\% Cl, $1.36-1.52$ ]; $\mathrm{P}<0.001)^{6}$. Similarly, lansoprazole $(\mathrm{a} \mathrm{PPI})$ is reported to promote amyloid-beta $(\mathrm{Ab})$ production ${ }^{7}$, the accumulation of which is central to one of the core hypotheses for the development of $A D^{12}$. More recently, a large population-based longitudinal study reported a 
more than a six-fold increased risk of $A D$ in individuals with inflammatory bowel disease (IBD) [HR: $6.19,95 \% \mathrm{Cl}: 3.31-11.57$ ], predicting over five-fold increased incidence across all forms of dementia ${ }^{9}$.

Taken together, evidence suggests comorbidity between AD and GIT disorders, although it is not clear whether GIT traits are risks for AD or vice versa. Regardless, these findings agree with the concept of the 'gut-brain' axis or the 'gastric mucosa-brain' relationship, which has been implicated in the association between GIT-related traits and central nervous system (CNS) disorders including depression and Parkinson's disease ${ }^{13-16}$. In support of a possible link between $A D$ and GIT traits, a recent animal model-based study indicates that intragastrointestinal accumulation of Ab may induce gastric function alteration, CNS amyloidosis, and subsequent $A D$-like dementia ${ }^{17}$. Comorbidity of $A D$ and GIT disorders may worsen the quality of life of sufferers while contributing significantly to increased healthcare costs.

Despite the increasing number of studies reporting an association between AD and GIT traits, the biological mechanism(s) underlying this association remains unclear. Moreover, contrasting evidence exists $9,18,19$, and many questions are unanswered. First, is there a riskincreasing association between AD and GIT disorders (including medicines commonly used for PUD, GERD, or gastritis-duodenitis)-PGM? This question assumes great importance in the face of contrasting evidence and longstanding debates on this subject ${ }^{18-20}$. Second, is there a causal relationship between $A D$ and GIT disorders (vertical pleiotropy)? Third, are there genetic components-e.g., single nucleotide polymorphism (SNPs), genes, and genomic loci-shared by AD and GIT disorders (biological pleiotropy)? Last, what biological pathways, processes, or mechanisms underlie any association between AD and GIT disorders? 
Large-scale genome-wide association studies (GWAS), identifying an increasing number of SNPs, genes, and susceptibility loci, have been conducted separately for AD and a range of GIT traits ${ }^{21-24}$. Findings from these GWAS provide compelling evidence for the roles of genetics in the aetiologies of AD and GIT disorders including PUD, PGM, gastritis-duodenitis, GERD, irritable bowel syndrome (IBS), diverticular disease, and IBD ${ }^{21-24}$. However, to the best of our knowledge, no study has leveraged the possible pleiotropy between AD and GIT disorders as a basis for discovering new shared SNPs, genes and/or susceptibility loci. Hence, it is unclear whether AD shares genetic susceptibility with any of these GIT disorders.

Moreover, studies assessing the mechanism(s) of association between AD and GIT disorders, based on the analysis of molecular genetic data, are lacking. We use a set of statistical genetics approaches in the analysis of well-powered GWAS data to comprehensively assess the genetic relationship between $A D$ and GIT disorders-PUD, GERD, PGM, IBS, gastritisduodenitis, diverticular disease, and IBD. The outcomes of this study have the potential to improve our understanding of the genetic architecture of AD and each of the GIT disorders, provide insights into their possible underlying biology, and characterise potential targets for further investigation.

\section{Results}

A schematic of the workflow for this study is presented in Figure 1. Also, the GWAS summary statistics analysed are summarised in Table 1 with further cohort-specific details provided in Supplementary Table 1. 
We first tested for SNP-level genetic overlap between AD and GIT disorders using the SNP effect concordance analysis (SECA) method ${ }^{25}$. Briefly, SECA performs a bi-directional analysis, assessing the effects of AD-associated SNPs (dataset 1) on each of GIT disorders (dataset 2) and vice versa. We found a significant concordance of SNP risk effects between the AD GWAS and each of the GERD, PUD, PGM, gastritis-duodenitis, IBS and diverticular disease GWAS, indicating that there is a strong genetic overlap between $A D$ and each of these disorders. Table 2 summarises the results of the primary test for the concordance of effects in which 144 SNP subsets were tested, with AD as P1 (dataset 1 ) and GERD as P2 (dataset 2). All these SNP subsets showed significant concordance of effects $(O R>1$ and $P<0.05)$ with a permuted P-value $\left(P_{\text {permuted }}\right)=9.99 \times 10^{-4} . A$ total of 26,963 linkage disequilibrium (LD)-independent SNPs $\left(r^{2}<0.1\right)$ were common to both the AD and GERD GWAS (at P $\left.1_{\text {GWAS-data }}=\mathrm{P} 2_{\text {GWAS-data }} \leq 1\right)$, $13,955(52 \%)$ of which exhibit significant effect concordance across the two GWAS (OR $=1.18$, $\left.P_{\text {Fisher's-exact }}=4.65 \times 10^{-11}\right)[$ Table 2].

As expected, a pattern of increasing strength of association between AD and GERD (measured using the OR values) was observed as the P-values for SNP subsets (P1 and P2) decreases. For example, at $A D\left(P 1_{\text {GWAS-data }}<0.05\right)$ and GERD $\left(P 2_{\text {GWAS-data }}<0.05\right)$, the proportion of SNP effect concordance increases to $58 \%\left(\mathrm{OR}=1.84, P_{\text {Fisher's-exact }}=1.74 \times 10^{-6}\right)$. In a reverse analysis (GERD as dataset 1 (P1) and AD as dataset 2 (P2)) using SECA, we also found all the 144 subsets of SNPs $\left(O R>1\right.$ and $\left.\mathrm{P}<0.05, \mathrm{P}_{\text {permuted }}=9.99 \times 10^{-4}\right)$ showing significant concordance of SNP risk effects across the two disorders [Supplementary Note I]. These results indicate that ADassociated SNPs are also associated with GERD, and vice versa-supporting evidence of highly significant genetic overlap between the two disorders. 

found significant genetic overlap between $A D$ and each of PGM, gastritis-duodenitis, diverticulosis and PUD. SECA similarly found a significant genetic overlap between AD and IBS, although the strength of association was comparatively less than for the other GIT disorders (Supplementary Note I). For AD and IBD, SECA revealed significant concordance of SNP risk effects when $A D$ was assessed (as dataset 1 ) against IBD (as dataset 2) [OR $>1$ and PGWAS-data $\left.<0.05, P_{\text {permuted }}=0.025\right]$ but not the other way around (Supplementary Note I).

\section{Genetic correlation between AD and GIT disorders}

We used the linkage disequilibrium score regression (LDSC) method to further assess and quantify the SNP-level genetic correlation between AD and GIT disorders (Table 3). The apolipoprotein $E(A P O E)$ region has a large effect on the risk of $A D$; hence, we excluded $A P O E$ and the 500 kilobase (kb) flanking region (hg19, 19:44,909,039-45,912,650) from our AD GWAS. Given the complex LD structure in the human major histocompatibility complex (MHC), we also excluded SNPs in the 26 to 36 megabase region of chromosome six from data. In analyses both with and without the APOE and MHC regions, LDSC reveals a significant genetic correlation between AD and several of the GIT traits (Table 3). $\left.\times 10^{-7}\right), \operatorname{PUD}\left(r_{g}=0.26, P=2.92 \times 10^{-4}\right), P G M\left(r_{g}=0.15, P=1.43 \times 10^{-4}\right)$, gastritis-duodenitis $\left(r_{g}\right.$ $\left.=0.19, \mathrm{P}=5.40 \times 10^{-3}\right)$, IBS $\left(r_{g}=0.16, P=2.36 \times 10^{-2}\right)$, and diverticular disease $\left(r_{g}=0.18, P=\right.$ $\left.1.59 \times 10^{-3}\right)$. These results are all consistent with our findings using SECA. Moreover, our results were based on the unconstrained genetic covariance intercept, hence the significance 
of our estimates may be conservative given the negligible, or complete absence of, sample overlap between the pairs of traits assessed.

Using LDSC, we did not find a significant genetic correlation between AD and IBD $\left(r_{g}=-0.05\right.$, $P=3.80 \times 10^{-1}$ ) [Table 3], a result that is partially consistent with our SECA findingshighlighting how SECA differs (a bidirectional assessment of the relationships) as well as complements LDSC. Table 3 summarises the findings of all these analyses. We also performed cross-trait LDSC analysis assessing the relationship between each of the GWAS included in this study (Supplementary Table 2). Notably, there was a non-significant relationship of IBD disorders (Supplementary Table 2).

\section{SNPs and loci shared by AD and GIT disorders}

Leveraging the significant genetic overlap and correlation as well as the substantial sample sizes of PGM, GERD and PUD, we performed cross-disorder meta-analyses of AD with each of SNPs/loci), which were also significantly associated with a GIT disorder, and vice versa, 
181

182

183

184

185

186

187

A total of 42 SNPs reached genome-wide significance $\left(P_{\text {meta-analysis }}<5 \times 10^{-8}\right)$ in the crossdisorder meta-analysis of AD and PGM GWAS (Supplementary Table 3). None of these 42 SNPs was genome-wide significant in the individual AD or PGM GWAS (before meta-analysis) $\left[\mathrm{P}_{\mathrm{GWAS} \text {-data }}>5 \times 10^{-8}\right]$ but they were at least nominally significant $\left(\mathrm{P}_{\mathrm{GWAS}-\mathrm{data}}<0.05\right)$ in each of the traits $\left(5 \times 10^{-8}<\mathrm{P}_{\text {GWAS-data }}<0.05\right)$. Of the 42 genome-wide significant SNPs, 11 were moderately independent $\left(r^{2}<0.6\right)$, from which we characterised seven lead SNPs at seven genomic loci $\left(r^{2}<0.1\right)$ [Table 4]. A search in the PhenoScanner ${ }^{26}$ (accessed on 04/05/2021), revealed that one of the 11 independent SNPs, rs11083749 (on chromosome 19q13.32, NECTIN2), has been reported for association with $A D$ at a genome-wide significant level. Our study provides evidence that this SNP and locus are equally associated with PGM given the substantial reduction in the GWAS meta-analysis P-value.

Of the remaining nine independent SNPs, at six genomic loci, none was previously found to be associated with AD, GERD, or PUD at a genome-wide level of significance, suggesting them to be novel SNPs and loci for the analysed traits (Table 4). Moreover, the results for m-value posterior probability and the binary effect P-value $(B E)$ indicate that all the identified SNPS and loci, except the 3p21.31 locus (SNPs rs709210 [ HYAL2] and rs7642934 [SEMA3F]), are associated with both AD and PGM. The m-value for each of the remaining SNPs was $>0.90$, predicting that they have effects in both GWAS (Table 4). Notably, the 3p21.31 locus (HYAL2 and SEMA3F), was subsequently identified to have effects both in AD and GIT-trait (based on the binary effect P-value results) in the meta-analysis of AD and PUD GWAS (Table 4).

We identified an additional 23 SNPs, at three independent loci $\left(r^{2}<0.1\right)$, that reached genome-wide suggestive association $\left(\mathrm{P}_{\text {meta-analysis }}<1 \times 10^{-5}\right.$ ) in the meta-analysis of $A D$ and 
PGM (supplementary Table 4). Of these, the rs33998678 SNP (at 16q22.1, IL34) is in high LD

204

205

206

207

208

209

210

211

212

213

214

215

216

217

$\left(r^{2}=0.91\right)$ with one of the genome-wide significant SNP loci ( $r$ 34644948, at 16q22.1, MTSS2, Table 4) identified in the meta-analysis of $A D$ and PGM. The finding, thus, supports the involvement of the locus (16q22.1, MTSS2) in both AD and GERD or PUD (traits represented by PGM GWAS). Similarly, the rs663576 (at 17q21.32, PHOSPHO1) is moderately correlated $\left(r^{2}=0.41\right)$ with another genome-wide significant SNP ( $r$ s2584662 at 17q21.33, PHB, Table 4), identified in the meta-analysis. This locus (17q21.33) was reproduced in the meta-analysis of AD and GERD (SNP rs2584662 near PHB), lending support for its involvement in AD and PUD or GERD. Notably, all the three loci reaching suggestive associations were predicted, using mvalue and the BE P-value methods, to have effects in both $A D$ and PGM.

\section{AD and GERD GWAS meta-analysis}

A meta-analysis of AD and GERD identified a total of 119 SNPs reaching genome-wide significant association ( $\mathrm{P}_{\text {meta-analysis }}<5 \times 10^{-8}$, Supplementary Table 5 ), none of which was previously genome-wide significant in the individual AD or GERD GWAS $\left(5 \times 10^{-8}<\right.$ PGWAS-data $_{\text {G }}$ $<0.05)$. From these, we characterised 11 independent SNPs $\left(r^{2}<0.6\right)$ and seven lead SNPs $\left(r^{2}<0.1\right)$ at seven genomic loci (Table 4). The identified loci included those implicated in the meta-analysis of $A D$ and PGM at a genome-wide level of significance $\left(P_{\text {meta-analysis }}<5 \times 10^{-8}\right)-$ 1p31.3, 3p21.31, 6p21.32, 17q21.33 and 19q13.32-and at a genome-wide suggestive association level $\left(P_{\text {meta-analysis }}<1 \times 10^{-5}\right)-16 q 22.1$ and $1 \mathrm{q} 32.2$.

Also, we found one (nearby) locus, 17q21.32 (SNP rs8067459, ZNF652, Table 4) reaching genome-wide significance in the AD and GERD meta-analysis. This locus was genome-wide suggestive in the AD vs PGM GWAS meta-analysis (SNP rs663576, 17q21.32, LD between rs663576 and rs8067459 $=0.86$ ), providing additional evidence for the locus being shared by 
AD and the GIT disorders (GERD and PUD). An additional 175 independent SNPs at 121 loci

227 reached a genome-wide suggestive association $\left(\mathrm{P}_{\text {meta-analysis }}<1 \times 10^{-5}\right.$ ) reproducing some of the genome-wide significant loci in the AD and PGM or the AD and GERD meta-analysis. The loci include 1p31.3 (rs2840677, PDE4B), 1q31.1 (rs10753964, BRINP3) and 1q32.2 (rs4147104, CD46) [Supplementary Table 6). Thus, the results support the loci being shared by AD and GERD. Other SNPs and loci reproduced at the suggestive association (or in high LD with identified loci) are highlighted in Supplementary Table 6.

\section{AD and PUD GWAS meta-analysis}

We identified 22 SNPs reaching genome-wide significance in the meta-analysis of AD and PUD GWAS ( $P_{\text {meta-analysis }}<5 \times 10^{-8}$, Supplementary Table 7). From these, we characterised seven independent SNPs at six genomic loci (Table 4) associated with both AD and PUD. Both the mvalue $(>0.90)$ and the BE methods predict that the identified SNPs and loci have effects in AD and PUD (Table 4). Of the loci identified in the $A D$ and PGM meta-analysis, four were replicated in the AD and PUD meta-analysis. Two of the four loci, the 19q13.32 ( $r$ s28363848 near BCL3), and the 6p21.32 (rs9270599, HLA-DRA), were replicated at a genome-wide level of significance, while the remaining two-rs709210, 3p21.31, $P_{(\mathrm{FE})}=5.24 \times 10^{-3}$, HYAL2; and rs6695557, 1p31.3, $P_{(\mathrm{FE})}=2.94 \times 10^{-4}, P D E 4 B-$ were replicated at a nominal level (significant reduction in P-value after AD and PUD meta-analysis, Supplementary Table 8). The SNP rs530324, at 8p21.1 (SCARA3, Table 4), identified in the AD and PUD meta-analysis, is in strong LD $\left(r^{2}=0.91\right)$ with another SNP ( $r$ 4732732, CLU) which reached a suggestive association for AD and PUD (Supplementary Table 9). The finding, thus, provides additional evidence for the involvement of the locus (8p21.1) in both AD and PUD. Supplementary Table 9 presents 24 
independent SNPs, at 21 genomic loci, reaching genome-wide suggestive association $\left(\mathrm{P}_{\text {meta- }}\right.$ analysis $<1 \times 10^{-5}$ ) for AD and PUD.

\section{Shared genomic regions}

251 Using a colocalization analysis in GWAS-PW ${ }^{27}$, we assessed shared genomic regions between $A D$ and each of PGM and GERD (Supplementary Table 10). The results of this analysis confirm that all the loci identified in the meta-analyses (except in chromosome 3 ) are shared by AD and the respective GIT traits (model 4 posterior probability [PPA 4] $>0.9$, Supplementary Table 10). While the findings also suggest that the causal variants might be different (in some of the loci-PPA $3<0.5$ ), we note that when variants in a locus are in strong $L D$, which is likely the case here, GWAS-PW is limited in its ability to correctly distinguish model 3 (PPA 3) from model 4 (PPA 4) ${ }^{27}$. Additional shared genomic regions, in chromosomes 1, 6, 16, 17 and 19 having PPA $4>0.90$ were identified for AD and the GIT traits (Supplementary Table 10). Also, we identified another locus on chromosome 17, having PPA $3>0.80$, and implicating the SNP rs2526380 (17q22, TSPOAP1) in both AD and GERD. The posterior probability that this SNP is a causal variant under model $3^{27}$ is high at 0.99 (Supplementary Table 10).

\section{Partial causal associations between AD and GIT disorders}

We assessed the causal relationship between AD (as the outcome variable) and GERD (as the exposure variable) using the two-sample Mendelian randomisation (MR) method. We found no evidence of a causal relationship between $A D$ and GERD, irrespective of the direction of the analysis (AD or GERD as the outcome or exposure variable) [Table 5]. For sensitivity testing, we implemented five additional models of MR analysis-MR-Egger, weighted median, simple mode, weighted mode and the MR-PRESSO (Mendelian Randomization Pleiotropy 
RESidual Sum and Outlier)-since a true finding will be consistent across the multiple

271

272

273

274

275

276

277

methods. Results of all these methods agree with those of the Inverse Variance Weighted (IVW) model supporting a lack of evidence for a causal association between AD and GERD (Table 5 and Supplementary Table 11). We carried out further MR analysis assessing AD against each of PUD, PGM, IBS, diverticular disease, and IBD. Findings similarly reveal no evidence for a causal relationship between $A D$ and each of the GIT-disorders assessed (Supplementary Note II, and Supplementary Table 11).

We also used the Latent Causal Variable (LCV) approach ${ }^{28}$ to test for a causal relationship between AD and each of the GIT disorders. The results of LCV suggest a partial causal influence of gastritis-duodenitis (genetic causal proportion $[\mathrm{GCP}]=-0.69, \mathrm{P}=0.0026$, Table 6), IBD (GCP $=-0.52, P=0.002319$, Table 6$)$ and lansoprazole $(G C P=-0.38, P=0.001129)$ on AD. The result was in the reverse direction for diverticular disease $(G C P=0.23, P=0.000272)$, suggesting a partial causal influence of $A D$ on this disorder.

\section{Gene-based association analysis}

Using a gene-based analysis of the SNPs that overlapped between the AD and PGM GWAS, we identified a total of 18,763 protein-coding genes for each of the traits. Applying a threshold P-value of $2.66 \times 10^{-6}$ (0.05/18763-Bonferroni correction for testing 18,763 genes), we identified 64 genome-wide significant $\left(P_{\text {gene }}<2.66 \times 10^{-6}\right)$ genes for AD (Supplementary Table 12), 75 for PGM (Supplementary Table 13), and 44 for GERD (Supplementary Table 14). Using the Fisher's Combined P-value (FCP) method, a total of 44 genome-wide significant ( $\mathrm{PFCP}_{\mathrm{F}}$ $<2.66 \times 10^{-6}$ ) genes shared by AD and PGM were identified, 11 of which were not previously significant in the individual AD or PGM GWAS (Supplementary Table 15). It is noteworthy that 
some of the identified $A D$ and PGM shared genes are in chromosomal locations found in our meta-analysis, including 1p31.3 (PDE4B), 3p21.31, (SEMA3F, HYAL2), 6p21.32 (HLA-DRA) and 19q13.32 (several apolipoprotein genes). We replicated a similar pattern of findings using the AD and the GERD GWAS (Supplementary Table 16).

\section{Biological pathways and mechanisms shared by AD and GIT disorders}

To identify significantly enriched biological pathways, mechanisms, and processes shared by $A D$ and GIT disorders, we performed pathway-based functional enrichment analyses in the g: Profiler platform ${ }^{29}$. These analyses enable us to functionally interpret genes overlapping between $A D$ and GIT disorders and can provide biological insight from their commonalities. First, we investigated genes overlapping $A D$ and GERD (at $P_{\text {gene }}<0.05, F C P<0.02$ ) and identified several biological pathways that were overrepresented (Supplementary Table 17, and Figure 2), implying they have a role in the mechanisms underlying both AD and GERD.

Pathways related to the metabolism, alteration, lowering or inhibition of lipids were significantly enriched for both traits (Supplementary Table 17). These included plasma lipoprotein assembly, remodelling, and clearance $\left(\mathrm{P}_{\text {adjusted }}=2.01 \times 10^{-3}\right)$, cholesterol metabolism $\left(\mathrm{P}_{\text {adjusted }}=4.99 \times 10^{-2}\right)$, plasma lipoprotein assembly $\left(\mathrm{P}_{\text {adjusted }}=3.45 \times 10^{-5}\right)$, and triglyceride-rich plasma lipoprotein particle $\left(P_{\text {adjusted }}=5.23 \times 10^{-9}\right)$, among others. Also, lipase inhibitors $\left(\mathrm{P}_{\text {adjusted }}=6.08 \times 10^{-3}\right)$ and the statin $(3-$ Hydroxy-3-methylglutaryl-coenzyme $\mathrm{A}$ reductase inhibitors) pathway $\left(\mathrm{P}_{\text {adjusted }}=3.99 \times 10^{-2}\right)$ were significantly enriched for $A D$ and GERD (Supplementary Table 17).

Pathways related to the immune system were also overrepresented for both AD and GERD as evidenced by the identification of immune or autoimmune-related disorders such as asthma 
$314\left(P_{\text {adjusted }}=3.53 \times 10^{-3}\right)$, systemic lupus erythematosus $\left(P_{\text {adjusted }}=7.88 \times 10^{-3}\right)$, and type I 315 diabetes mellitus $\left(P_{\text {adjusted }}=2.47 \times 10^{-2}\right)$. Other immune-related pathways identified include 316 intestinal immune network for IgA production $\left(P_{\text {adjusted }}=4.07 \times 10^{-2}\right)$, programmed cell death 317 protein $1(P D-1)$ signalling $\left(P_{\text {adjusted }}=5.24 \times 10^{-3}\right)$, translocation of ZAP-70 to immunological 318 synapse $\left(\mathrm{P}_{\text {adjusted }}=2.44 \times 10^{-3}\right)$, and interferon-gamma signalling pathways $\left(\mathrm{P}_{\text {adjusted }}=2.45 \times\right.$ $31910^{-2}$ ) [Supplementary Table 17].

Following enrichment mapping and auto-annotation, the identified biological pathways were clustered into six themes of biological mechanisms, namely: 'lipoprotein particle clearance,' 'receptor signalling pathway,' 'side membrane vesicle and cell adhesion,' 'peptide antigen binding,' 'intestinal immune network,' and 'interferon-gamma signalling' (Figure 2). Moreover, a pathway-based analysis using genes that were overlapping between the AD and PGM GWAS (at $P_{\text {gene }}<0.05$ ) replicated some of the pathways identified for AD and GERD, including 'plasma lipoprotein assembly, remodelling, and clearance' $\left(P_{\text {adjusted }}=3.01 \times 10^{-4}\right)$, 'peptide antigen binding' $\left(\mathrm{P}_{\text {adjusted }}=2.28 \times 10^{-3}\right)$, and 'triglyceride-rich plasma lipoprotein particle' $\left(P_{\text {adjusted }}=6.60 \times 10^{-8}\right)$ [Supplementary Table 18, and Supplementary Note III]. Figure 3 summarises the relationships of these mechanisms and how they may apply in AD and GIT disorders.

\section{Discussion}

We present the first comprehensive assessment of the genetic relationship between AD and GIT disorders by analysing large scale GWAS summary data using multiple statistical genetics approaches. We found a significant genetic overlap and correlation between $A D$ and each of GERD, PUD, PGM (medications for PUD or GERD), gastritis-duodenitis, IBS, and diverticular 
disease. These results support evidence of shared genetic susceptibility between AD and these GIT traits. Also, we identified several independent SNPs, susceptibility loci, genes and biological pathways shared by AD and each of GERD and PUD. These findings not only confirm the results of previous observational studies ${ }^{5-11}$ which have suggested a co-occurring association of AD with GIT disorders but also provide novel insights into the mechanisms underlying the observed associations.

The use of multiple, complementary statistical genetic approaches enables a comprehensive analysis of the genetic associations between AD and GIT disorders and is a major strength of this study. Also, we analysed well-powered GWAS data, meaning our findings are not affected by the small sample sizes, possible reverse causality, or confounders that conventional observational studies often suffer from. One potential limitation of our data is that the GWAS for $A D$ combined clinically diagnosed cases of $A D$ with proxies (AD-by-proxy-individuals whose parents were diagnosed with $A D$ ). Given the high correlation between the GWAS with and without the 'AD-by-proxy' cases ${ }^{21}$, we argue that combining them is valid, especially for sample size improvement. However, likely, some of the proxy samples will not develop AD. This observation may influence the significance of our causality estimates. Hence, causal associations between AD and GIT disorders may not be ruled out.

In contrast to the strong positive genetic correlation between AD and the GIT traits examined, we did not find a significant genetic correlation between AD and IBD using LDSC, which may be due to the relatively small number of cases in the IBD GWAS. Supporting this premise, SECA revealed a significant genetic overlap between the disorders when AD was assessed as dataset 1 against IBD as dataset 2, but not the other way around. The AD GWAS has a much larger case and total sample size and therefore provides a more robust association on which 
to condition (select independent) SNPs for concordance analysis. Alternatively, IBD may have a different mechanism from the rest of the GIT disorders and is not associated with AD. This position is suggested by the non-significant genetic correlation of IBD with the other GIT disorders, except IBS. Given these findings, other results in our study (e.g., LCV finding) potentially supporting IBD mechanisms, and the highly significant association between AD and IBD reported in a previous study ${ }^{9}$, future studies need to further investigate the relationship between $A D$ and IBD using more powerful IBD GWAS.

Evidence of significant genetic overlap and correlation reflects not only shared genetic aetiologies (biological pleiotropy) but also suggests a possible causal association between AD and the GIT traits (vertical pleiotropy). Using LCV, we detected a partial causal association between $A D$ and each of gastritis-duodenitis, lansoprazole, IBD and diverticular disease. However, when we attempted to reproduce findings for gastritis-duodenitis using another GWAS (also for lansoprazole using PGM), this causal association was not evident; hence, a reassessment of the inconclusive LCV results, in future studies, may be warranted. Conversely, MR analyses did not provide evidence for a significant causal relationship between AD and GIT disorders, indicating that shared genetics and biological pathways best explain the association between AD and these GIT disorders.

We performed GWAS meta-analysis to identify shared SNPs and susceptibility loci, leveraging the larger sample sizes of the PGM, GERD and PUD GWAS, and the significant pleiotropy identified between $A D$ and each of these GIT traits. Our meta-analysis of the AD and PGM GWAS identified seven shared independent loci reaching genome-wide significance for association with both traits. Several of these loci were also identified in meta-analyses of $A D$ with GERD and PUD, providing strong support for their involvement in both AD and GIT 
disorders. Results from 'm-value,' binary effect ${ }^{30}$, and GWAS-PW ${ }^{27}$ methods, overall, robustly support these results. Moreover, many of the loci, including $1 \mathrm{p} 31.3$ (PDE4B), 3p21.31 (SEMA3F, HYAL2), 6p21.32 (HLA-DRA), and 19q13.32 (APOE, APOC2, ERCC2, BCL3, and KLC3 genes) were replicated in our gene-based association analyses. Following a search in PhenoScanner, six of these loci-1p31.3 (PDE4B), 1q32.2 (CD46), 3p21.31 (SEMA3F, HYAL2), 6p21.32 (HLA-DQA2, HLA-DRA), 16q22.1 (MTSS2), 17q21.33 (PHB)-have not previously been reported, at a genome-wide level of significance, for AD, GERD, or PUD, indicating they are putative novel loci associated with these traits. The remaining locus (19q13.32), harbouring the $A P O E$ gene, has a well-established association with $A D$, and our results suggest it is also involved in these GIT disorders. Consistent with this position, the APOE genotype is associated with the gut microbiome ${ }^{31}$, and IBD 32,33 .

Notably, the independent SNP rs12058296 (1p31.3), which reached genome-wide significance for both $A D$ and PGM, mapped to the phosphodiesterase $4 B$ (PDE4B) gene. Inhibition of $P D E 4 B$ (or its subtypes) has shown promise as a treatment for inflammatory diseases ${ }^{34-37}$. Indeed, consistent recent evidence supports the potent anti-inflammatory, procognitive, neuro-regenerative, and memory-enhancing properties of PDE4 inhibitors (PDE4B, in particular ${ }^{38}$ ), making them plausible therapeutic targets for $\mathrm{AD}^{36,37}$ and GIT disorders ${ }^{35}$. Other identified independent genome-wide significant SNPs and loci mapped to genes including CD46, SEMA3F, HLA-DRA, MTSS2, PHB, and APOE. The CD46 gene is a complement regulator which is bactericidal to Helicobacter $(H)$ pylori ${ }^{39}$ and was also recently identified to be associated with $A D$ in a transcriptome analysis ${ }^{40}$, making it a plausible candidate in both $A D$ and GIT disorders. 
Using pathway-based analyses, we identified biological pathways, mechanisms and processes significantly enriched for AD and GIT disorders. Notably, lipid-related, and autoimmune pathways were overrepresented. There is a close link between autoimmunity and lipid abnormalities ${ }^{41}$, and our findings highlight abnormal lipid profiles as risks for AD and GIT disorders, consistent with findings in previous studies ${ }^{42-46}$. In $A D$, for example, hypercholesterolemia is believed to increase the permeability of the blood-brain barrier system, facilitating the entry of peripheral cholesterol into the CNS, and resulting in abnormal cholesterol metabolism in the brain ${ }^{42,43}$. Amyloidogenesis, alteration of the amyloid precursor protein degradation, accumulation of $A b$, and subsequent cognitive impairment have all been linked with elevated cholesterol in the brain ${ }^{43,47-49}$. While the exact roles of lipids in GIT disorders are unclear, H. pylori is believed to cause or worsen abnormal serum lipid profiles through chronic inflammatory processes, and eradication of the infection enhances lipid homeostasis 45,46 .

The mechanisms of association between $A D$ and lipid dysregulation relate to the 'gut-brain axis', alterations in GIT microbiota and the immune system ${ }^{43}$. This observation is consistent with our findings, revealing the potential of lipid-lowering therapies such as lipase inhibitors and statins (identified in our study) for the treatment of AD and GIT disorders or their comorbidity. Lipase inhibitors such as orlistat prevent intestinal dietary lipid absorption, thereby decreasing total plasma triglycerides and cholesterol levels ${ }^{50,51}$, making them a preferred pharmacological treatment for obesity ${ }^{50}$. The acknowledged connection between $A D$, lipid dysregulation and the 'gut-brain axis' ${ }^{43}$, supports the potential utility of lipase inhibitors in AD. Other lipases, including monoacylglycerol, diacylglycerol, and lipoprotein 
lipases are involved in AD pathology, and can also effectively be inhibited by orlistat ${ }^{51}$. Thus, we hypothesise that lipase inhibitors may be promising in comorbid AD and GIT disorders.

Statins (cholesterol-lowering medications) are also therapeutically beneficial in AD and GIT disorders ${ }^{52-56}$. Evidence indicates that statins possess anti-inflammatory, immunemodulating and gastroprotective properties 52,53 , and their active use is associated with a significant PUD risk reduction ${ }^{52}$, and $H$. pylori eradication ${ }^{54}$. Further, statins improve cognitive ability and reduce neurodegeneration risks, making them potentially beneficial in $A D^{55,56}$. However, there is (controversial) evidence suggesting a paradoxical predisposition to reversible dementia for statins ${ }^{55,56}$. While this finding has been challenged ${ }^{55}$, it highlights a clear need to identify $A D$ patients for whom statins will be beneficial, consistent with the model of personalised health. Hence, we hypothesise that statins may be beneficial in individuals with comorbid AD and GIT disorders.

Our findings have implications for practice and further studies. First, results highlighting lipidrelated mechanisms support the roles of abnormal lipid profiles in the aetiologies of the disorders, which may be potential biomarkers for AD and GIT disorders (or their comorbidity). Second, our findings underscore the importance of lipid homeostasis. The dietary approach is one effective non-pharmacologic approach for the management of hyperlipidaemia, and overall, this is consistent with findings in this study. Indeed, adherence to a 'Mediterranean' diet (low in lipids) is recognised as beneficial both in AD ${ }^{57}$ and GIT disorders ${ }^{58}$. Thus, a recommendation for healthy diets, early in life, may form part of the lifestyle modifications for preventing $A D$ and GIT disorders. Third, our study identifies lipase inhibitors and statin pathways in the mechanisms of AD and GIT disorders which may be a potential therapeutic avenue to explore in the disorders. Hence, we hypothesise that individuals with comorbid AD 
and GIT traits may gain benefits from these therapies. Fourth, our study implicates the PDE4B,

450

451

452

453

454

455

456

457 and given the evidence in the literature ${ }^{35-38}$, we propose that treatment targeted at its inhibition may be promising in comorbid AD and GIT traits. Future studies, including randomised control trials, are needed to test these hypotheses. Lastly, the finding of significant genetic overlap between AD and GIT disorders suggests that it would be beneficial for healthcare providers to test for GIT disorders in individuals presenting with signs or symptoms of impaired cognition and vice versa, to improve early detection.

In summary, this study provides a novel insight into the relationship between AD and GIT disorders, implicating shared genetic susceptibility in the mechanisms of their association. To our knowledge, this is the first study to comprehensively assess these relationships using statistical genetics approaches. Overall, these findings advance our understanding of the genetic architecture of AD and GIT disorders. The shared genetic components and biological pathways identified are important targets for further investigation in AD and each of the GIT disorders.

\section{Methods}

\section{Datasets}

The GWAS data utilised in the present study were sourced from publicly available GWAS databases and repositories and are summarised in Supplementary Table 1. The recently published 'clinically diagnosed $A D$ and AD-by-proxy' ${ }^{21}$, with a large sample size, was used as our AD GWAS data. GIT traits including gastritis-duodenitis, GERD, PUD, IBS, diverticular disease, and IBD were assessed against $A D$, and the GWAS for these traits were obtained through the GWAS Atlas ${ }^{24}$ or the recently published GIT GWAS ${ }^{22}$. Additionally, we utilised a 
471 well-characterised GWAS for GERD, which combined datasets from the UK Biobank and the

472 QSKIN study 23 (https://figshare.com/articles/GERD_GWAS_summary/8986589), and

473 gastritis-duodenitis

474 (ftp://share.sph.umich.edu:21/UKBB_SAIGE_HRC/PheCode_535_SAIGE_MACge20.txt.vcf.gz

475 ). GWAS with a large sample size for medication use in PUD and GERD (PGM) are available and we utilised these as proxies to examine the relationship between AD and GIT disorders

477 (PUD and GERD, in particular). A comprehensive description of the quality control procedures 478 for each of the GWAS data and their analysis has previously been reported in the 479 corresponding publications (Supplementary Table 1).

\section{SNP effect concordance analysis (SECA)}

481 We used the standalone version of the SECA software pipeline 482 (https://sites.google.com/site/qutsgel/software/seca-local-version) to perform SNP-level genetic overlap assessment and statistical tests between AD and GIT disorders. A detailed description of the SECA software and methods has been published ${ }^{25}$. Briefly, SECA accepts a pair of GWAS data (dataset 1 and dataset 2) as input and performs a range of analyses to determine whether there is a genetic overlap between a pair of traits. First, we carried out quality control to exclude all non-rsID(s) and duplicate variants in dataset 1 , align SNP effects to the same effect allele across dataset 1 and dataset 2 and perform a P-value informed test for linkage disequilibrium (LD) clumping in the dataset 1 using PLINK ${ }^{59}$. Second, SECA partitions independent SNPs resulting from LD clumping (at $r^{2}<0.1$ ) into 12 subsets of SNPs according to the P-value for dataset 1 as follows: $\mathrm{P} 1 \leq(0.01,0.05,0.1,0.2,0.3,0.4,0.5,0.6$, $0.7,0.8,0.9,1.0)$. SECA subsequently performs Fisher's exact tests (FT) to assess the presence of excess SNPs in which the direction of effects is concordant across dataset 1 and dataset 2 
(that is, for the corresponding P-value derived 12 subsets of SNPs associated in dataset 2, P2). Hence, a total of 144 SNP subsets (a 12 by 12 matrix from dataset 1 and dataset 2) was assessed for SNP effect concordance. In the present study, we first assessed AD GWAS as dataset 1 and each of the GIT disorders as dataset 2. For comparison, we also assessed each of the GIT disorders as dataset 1 against AD as dataset 2. Thus, using SECA, we assessed the effects of AD-associated SNPS on each of GIT disorders and vice versa. This is an important analysis step to account for instances where SNPs that are strongly associated with AD do not affect GIT disorder traits and vice versa.

\section{Linkage disequilibrium score regression analysis (LDSC)}

LDSC assesses and distinguishes the contributions of polygenicity, sample overlaps, and population stratification to the heritability and genetic correlation between traits ${ }^{60}$. In the present study, we performed LDSC analysis using the standalone version of the software and by following the procedures provided by the program developer (https://github.com/bulik/ldsc). We conducted cross-trait bivariate LDSC to estimate the genetic correlation between AD and each of the GIT disorders assessed in this study. To avoid a potential bias from residual confounding, we did not constrain the genetic covariance intercepts in any of our genetic correlation analyses; this practice may be conservative and reduce the significance of correlation estimates in instances where there is no sample overlap between the pair of traits being assessed.

\section{GWAS Cross-disorder meta-analysis}

514 GWAS meta-analysis pools the results of GWAS data, thereby increasing the sample sizes and augmenting the detection of genetic variants with small to modest effect sizes. In the present 
study, we used the GWAS meta-analysis method of pooling AD GWAS with each of the GIT traits (cross-disorder or cross-trait meta-analysis). We used two models of meta-analysis: the

518 Fixed Effect (FE), and the modified Random Effect (RE2) ${ }^{61}$ models, implemented in the METASOFT software (http://genetics.cs.ucla.edu/meta/). The FE model estimates the FE P-

520 value using the inverse-variance weighted method, which assumes that the AD and each of 521 the GIT disorders' GWAS are assessing the same (fixed) effect. The presence of effect

522 heterogeneity is a limitation of the model. On the other hand, by estimating P-values using the modified random-effects, the RE2 model ${ }^{61}$ allows for differences in SNP effects and the method is powerful in the presence of SNP effect heterogeneity. Furthermore, given the possibility of slight sample overlap between the AD GWAS and the GWAS for some of the GIT traits, we utilised an additional meta-analysis model, the RE2C model (modified Random Effect Correlation model) ${ }^{62}$. This method is suitable for GWAS meta-analysis of correlated statistics ${ }^{62}$. In the present study, we meta-analysed SNPs overlapping AD and each of the GIT disorders using the FE, and the RE2 models complementarily. In the meta-analyses for AD and GERD (genetic covariance intercept $=0.0133$, se $=0.005$ ) and $A D$ and PGM (genetic covariance intercept $=0.0135$, se $=0.006)$, we utilised the RE2C as an additional model to account for the slight sample overlap between the respective pairs of traits.

\section{Genomic loci characterisation}

534 Using the outputs of our cross-trait meta-analyses for AD and each of the GIT disorders, we carried out some downstream analyses including functional annotation of SNPs, and genomic

536 loci characterisation in line with practice in previous studies ${ }^{14,63,64}$. Briefly, SNPs that were not genome-wide significant in the individual AD and GIT disorder GWAS, but which reached genome-wide significance following the meta-analysis were identified. From these, we 
characterised independent SNPs at $r^{2}<0.6$, and lead SNPs at $r^{2}<0.1$. We defined the genomic

540 locus as the region within $250 \mathrm{~kb}$ of each lead SNP. We assigned lead SNPs within this region to the same locus, meaning two or more lead SNPs may be present in one locus. Several of these downstream analyses were performed in the FUMA software (an online platform) ${ }^{63}$.

\section{Assessment using the posterior probability (m-value) method}

To identify loci shared by AD and GIT disorders, we performed a further analysis using the posterior probability (m-value) method and the complementary binary effects (BE) P-value estimates ${ }^{30}$. Briefly, cross-study information was utilised in estimating the $\mathrm{m}$-value to predict whether a SNP or locus has effects in each of the studies meta-analysed, particularly in the presence of effect heterogeneity ${ }^{30}$. M-value ranges from 0 to 1 , where a value $>0.9$ predicts that effect exists for the SNP or locus in the study (e.g., AD GWAS or PUD GWAS). On the other hand, an m-value $<0.1$ predicts that an effect does not exist in the study. M-values from 0.1 to 0.9 predict an ambiguous effect. We used these methods to verify whether the SNPs or loci identified in our GWAS meta-analysis have effects (shared) by the two traits under investigation, especially where the test for heterogeneity was significant. We interpreted the results of the $\mathrm{m}$-value alongside the BE P-value. It is expected that, where effects exist in both traits being assessed, the BE P-value estimates will be less than the P-value for the respective GWAS. We implemented the BE and m-value methods alongside the FE, and the RE2 metaanalysis models using the METASOFT software.

\section{Pairwise GWAS analysis}

We performed co-localisation analysis utilising the pairwise GWAS (GWAS-PW) method ${ }^{27}$ to further assess the regions in the genome shared by AD and GIT-disorders. Briefly, GWAS-PW 
software implements the Bayesian pleiotropy association test and identifies genomic regions

562 that influence a pair of correlated traits ${ }^{27}$. We used this method to assess whether the loci reaching genome-wide significance in our GWAS meta-analyses were truly shared by AD and the GIT disorders. Also, we investigated other shared genomic regions which may not have been found in the GWAS meta-analysis. We combined the summary data for AD with the data for each of the GIT disorders and estimated the posterior probability of association (PPA) of a genomic region using the GWAS-PW software. We modelled four PPAs: i) that a genomic region is associated with AD only (PPA-1), ii) that a genomic region is associated with the GIT trait only (PPA-2), iii) that a genomic region is associated with both AD and the GIT trait (PPA3), and iv) that a specific genomic region is associated with both AD and the GIT trait but through separate causal variants (PPA-4) ${ }^{27}$.

\section{Causal relationships assessment}

573 Using Mendelian randomisation (MR) ${ }^{65}$ analysis methods, we examine the potential causal association between $A D$ and each of the GIT disorders assessed in this study. Mimicking randomised control trials (RCTs), MR analysis incorporates genetics into epidemiological study designs to assess causality ${ }^{65}$. In the present study, we used the two-sample MR method

577 (https://mrcieu.github.io/TwoSampleMR/articles/introduction.html) for a bidirectional association assessment between $A D$ and each of the GIT disorders. Independent $\left(r^{2}<0.001\right)$ genome-wide significant SNPs $\left(P<5 \times 10^{-8}\right)$ associated with AD (as the exposure variable) were utilised as instrumental variables (IVs) and assessed against each of the GIT disorders' GWAS (outcome variables) analysed in this study. 
Reversing the direction of analysis, independent SNPs robustly associated with each of the GIT disorders' GWAS (exposure variable) were similarly utilised as IVs and assessed against $A D$ (as the outcome variable). We used the inverse variance weighted (IVW) model of MR as the primary method for causal association assessment, and for validity testing, we performed a heterogeneity test (Cochran's Q-test), a 'leave-one-out' analysis, a horizontal pleiotropy check (MR-Egger intercept) and individual SNP MR analyses. Also, we used other MR analysis models including the weighted mode, simple mode, MR-Egger, weighted median ${ }^{66,67}$, and the 'Mendelian randomisation pleiotropy residual sum and outlier' (MR-PRESSO) ${ }^{68}$ methods for sensitivity testing. All MR analyses were performed in R (4.0.2).

We performed an additional assessment of the causal or partial causal association between $A D$ and each of the GIT disorders using the Latent Causal Variable (LCV) method ${ }^{28}$. LCV estimates causality proportion (GCP) ranging from -1 to 1 where a value close to 1 indicates a potential causal association between two traits in the forward direction and -1 in the backward direction ${ }^{28}$. This analysis was performed in the online platform of the CTG virtual laboratory (https://vl.genoma.io/analyses/lcv) ${ }^{28,69}$.

\section{Gene-based association analysis}

We performed gene-based association analyses to identify genome-wide significant genes shared by both $A D$ and each of the GIT disorders assessed in this study. This analysis complements the SNP-based studies. However, beyond the SNP-level analysis, gene-based association analysis provides greater power for identifying genetic risk variants since it aggregates the effects of multiple SNPs, and it is generally not limited by small effect sizes or correlations among SNPs. Moreover, genes are more closely related to biology than SNPS, 
604

605

606

607

608

609

610

611

612

613

614

615

616

617

618

619

620

621

622

623

624

625

meaning gene-level analysis can offer better insights into the underlying biological mechanisms of complex traits.

In the present study, we carried out gene-based association analysis separately for AD and each of the GIT disorders using the MAGMA software, implemented in FUMA ${ }^{63}$. Based on the results of the gene-based analysis, we identified genome-wide significant genes for each of the traits. Also, using the Fisher Combined P-value (FCP) method, we identified genes shared by $A D$ and each of the GIT traits.

\section{Pathway-based functional enrichment analysis}

For a better understanding of the potential biological mechanisms underlying the relationship of AD with GIT disorders, we carried out pathway-based functional enrichment analyses using the online platform of the g:GOst tool in the g-profiler software ${ }^{29}$. The analysis enables us to functionally interpret genes overlapping $A D$ and GIT disorders. We included genes that were overlapping between $A D$ and each of GERD and PGM at $\mathrm{P}_{\text {gene }}<0.05$ (FCP $\left.<0.02\right)$ in this analysis, and followed established protocols ${ }^{70}$. Functional category term sizes were restricted to values from 5 to $350^{70}$. For multiple testing corrections, we applied the default ' $\mathrm{g}$ : SCS algorithm' recommended in the protocol ${ }^{70}$ and reported the significantly enriched biological pathways at the multiple testing adjusted $\mathrm{P}$-value $\left[\mathrm{P}_{\text {adjusted }}\right]<0.05$.

\section{Data availability}

The GWAS summary data for AD and GIT disorders utilised in the present study are available in the public domain and can be accessed using the links provided herewith:

AD GWAS: https://ctg.cncr.nl/documents/p1651/AD_sumstats_Jansenetal_2019sept.txt.gz, PUD: https://cnsgenomics.com/data/wu_et_al_2021_nc/1_PUD_summary 
GERD: https://figshare.com/articles/GERD_GWAS_summary/8986589, Gastritis-duodenitis:

627

628

629

630

631

632

633

634

635

636

637

638

639

640

641

642

643

644 645 646 647

648

649

\section{8} ftp://share.sph.umich.edu:21/UKBB_SAIGE_HRC/PheCode_535_SAIGE_MACge20.txt.vcf.gz, IBS: https://cnsgenomics.com/data/wu_et_al_2021_nc/4_IBS_summary, PGM: https://cnsgenomics.com/data/wu_et_al_2021_nc/3_PGM_summary, IBD: https://cnsgenomics.com/data/wu_et_al_2021_nc/5_IBD_summary, IBD: ftp://ftp.sanger.ac.uk/pub/consortia/ibdgenetics/iibdgc-trans-ancestry-filteredsummary-stats.tgz, Diverticular disease:

ftp://share.sph.umich.edu:21/UKBB_SAIGE_HRC/PheCode_562_SAIGE_MACge20.txt.vcf.gz

\section{Acknowledgements}

This research was supported through funding to SML from the National Health and Medical Research Council (Australia; APP1161706, APP1191535). We gratefully appreciate UKB (through QSKIN study, and GWAS Atlas) for providing open access to the GWAS summary data utilised in this study.

(1)

1

2

643

4

(1) 


\section{References}

651

6521

653

654

655

656

657

658

659

660

661

662

663

664

665

666

667

668

669

670

671

672

673

674

675

676

677

678

679

680

681

682

683

684

685

686

687

688

689

690

691

692

693

694

695

696

697

698

Prince, M. J. World Alzheimer Report 2015: the global impact of dementia: an analysis of prevalence, incidence, cost and trends. (Alzheimer's Disease International, 2015).

2 Cummings, J., Lee, G., Ritter, A., Sabbagh, M. \& Zhong, K. Alzheimer's disease drug development pipeline: 2019. Alzheimer's \& Dementia: Translational Research \& Clinical Interventions 5, 272-293 (2019).

3 Takizawa, C., Thompson, P. L., van Walsem, A., Faure, C. \& Maier, W. C. Epidemiological and economic burden of Alzheimer's disease: a systematic literature review of data across Europe and the United States of America. J Alzheimers Dis 43, 1271-1284, doi:10.3233/jad-141134 (2015).

4 Levey, A. I. Progress with Treatments for Alzheimer's Disease. New England Journal of Medicine 384, 1762-1763, doi:10.1056/NEJMe2103722 (2021).

5 Momtaz, Y. A., Hamid, T. A. \& Ibrahim, R. Gastritis May Boost Odds of Dementia. American Journal of Alzheimer's Disease \& Other Dementiasr 29, 452-456, doi:10.1177/1533317513518654 (2014).

6 Gomm, W. et al. Association of proton pump inhibitors with risk of dementia: a pharmacoepidemiological claims data analysis. JAMA neurology 73, 410-416 (2016).

7 Badiola, N. et al. The proton-pump inhibitor lansoprazole enhances amyloid beta production. PloS one 8, e58837 (2013).

8 Jiang, C., Li, G., Huang, P., Liu, Z. \& Zhao, B. The gut microbiota and Alzheimer's disease. Journal of Alzheimer's Disease 58, 1-15 (2017).

9 Zhang, B. et al. Inflammatory bowel disease is associated with higher dementia risk: a nationwide longitudinal study. Gut 70, 85-91 (2021).

10 Chen, C.-H., Lin, C.-L. \& Kao, C.-H. Irritable Bowel Syndrome Is Associated with an Increased Risk of Dementia: A Nationwide Population-Based Study. PLOS ONE 11, e0144589, doi:10.1371/journal.pone.0144589 (2016).

11 Doulberis, M. et al. Alzheimer's disease and gastrointestinal microbiota; impact of Helicobacter pylori infection involvement. Int J Neurosci 131, 289-301, doi:10.1080/00207454.2020.1738432 (2021).

12 Selkoe, D. J. \& Hardy, J. The amyloid hypothesis of Alzheimer's disease at 25 years. EMBO molecular medicine 8, 595-608, doi:10.15252/emmm.201606210 (2016).

13 Kim, S. Y., Min, C., Oh, D. J. \& Choi, H. G. Reciprocal association between depression and peptic ulcers: Two longitudinal follow-up studies using a national sample cohort. Scientific Reports 10, 1749, doi:10.1038/s41598-020-58783-0 (2020).

14 Adewuyi, E. O. et al. Genetic analysis of endometriosis and depression identifies shared loci and implicates causal links with gastric mucosa abnormality. Human Genetics 140, 529-552, doi:10.1007/s00439-020-02223-6 (2021).

15 Menozzi, E., Macnaughtan, J. \& Schapira, A. H. V. The gut-brain axis and Parkinson disease: clinical and pathogenetic relevance. Annals of Medicine 53, 611-625, doi:10.1080/07853890.2021.1890330 (2021).

16 Kowalski, K. \& Mulak, A. Brain-Gut-Microbiota Axis in Alzheimer\&amp;rsquo;s Disease. J Neurogastroenterol Motil 25, 48-60, doi:10.5056/jnm18087 (2019).

17 Sun, Y. et al. Intra-gastrointestinal amyloid- $\beta 1-42$ oligomers perturb enteric function and induce Alzheimer's disease pathology. The Journal of physiology 598, 4209-4223 (2020).

18 Khan, M. A. et al. No Association Linking Short-Term Proton Pump Inhibitor Use to Dementia: Systematic Review and Meta-analysis of Observational Studies. Official journal of the American College of Gastroenterology / ACG 115, 671-678, doi:10.14309/ajg.0000000000000500 (2020). 
19 Zhang, Y. et al. Proton pump inhibitors use and dementia risk: a meta-analysis of cohort studies. European Journal of Clinical Pharmacology 76, 139-147, doi:10.1007/s00228-01902753-7 (2020).

20 Wu, C.-L. et al. Acid suppressants use and the risk of dementia: A population-based propensity score-matched cohort study. PLOS ONE 15, e0242975, doi:10.1371/journal.pone.0242975 (2020).

21 Jansen, I. E. et al. Genome-wide meta-analysis identifies new loci and functional pathways influencing Alzheimer's disease risk. Nature genetics 51, 404-413 (2019).

22 Wu, Y. et al. GWAS of peptic ulcer disease implicates Helicobacter pylori infection, other gastrointestinal disorders and depression. Nature Communications 12, 1146, doi:10.1038/s41467-021-21280-7 (2021).

23 An, J. et al. Gastroesophageal reflux GWAS identifies risk loci that also associate with subsequent severe esophageal diseases. Nature Communications 10, 4219, doi:10.1038/s41467-019-11968-2 (2019).

24 Watanabe, K. et al. A global overview of pleiotropy and genetic architecture in complex traits. Nat Genet 51, 1339-1348, doi:10.1038/s41588-019-0481-0 (2019).

25 Nyholt, D. R. SECA: SNP effect concordance analysis using genome-wide association summary results. Bioinformatics 30, 2086-2088 (2014).

26 Staley, J. R. et al. PhenoScanner: a database of human genotype-phenotype associations. Bioinformatics 32, 3207-3209, doi:10.1093/bioinformatics/btw373 (2016).

27 Pickrell, J. K. et al. Detection and interpretation of shared genetic influences on 42 human traits. Nature genetics 48, 709 (2016).

28 O'Connor, L. J. \& Price, A. L. Distinguishing genetic correlation from causation across 52 diseases and complex traits. Nature Genetics 50, 1728-1734, doi:10.1038/s41588-018-0255-0 (2018).

29 Raudvere, U. et al. g:Profiler: a web server for functional enrichment analysis and conversions of gene lists (2019 update). Nucleic Acids Research 47, W191-W198, doi:10.1093/nar/gkz369 (2019).

30 Han, B. \& Eskin, E. Interpreting Meta-Analyses of Genome-Wide Association Studies. PLOS Genetics 8, e1002555, doi:10.1371/journal.pgen.1002555 (2012).

31 Parikh, I. J. et al. Murine Gut Microbiome Association With APOE Alleles. Frontiers in Immunology 11, doi:10.3389/fimmu.2020.00200 (2020).

32 Al-Meghaiseeb, E. S. et al. Genetic association of apolipoprotein E polymorphisms with inflammatory bowel disease. World journal of gastroenterology: WJG 21, 897 (2015).

33 Glapa-Nowak, A. et al. Apolipoprotein E variants correlate with the clinical presentation of paediatric inflammatory bowel disease: A cross-sectional study. World J Gastroenterol 27, 1483-1496, doi:10.3748/wjg.v27.i14.1483 (2021).

$34 \mathrm{Li}, \mathrm{H}$., Zuo, J. \& Tang, W. Phosphodiesterase-4 Inhibitors for the Treatment of Inflammatory Diseases. Front Pharmacol 9, 1048-1048, doi:10.3389/fphar.2018.01048 (2018).

35 Spadaccini, M., D'Alessio, S., Peyrin-Biroulet, L. \& Danese, S. PDE4 Inhibition and Inflammatory Bowel Disease: A Novel Therapeutic Avenue. Int J Mol Sci 18, 1276, doi:10.3390/ijms18061276 (2017).

36 Richter, W., Menniti, F. S., Zhang, H.-T. \& Conti, M. PDE4 as a target for cognition enhancement. Expert Opin Ther Targets 17, 1011-1027, doi:10.1517/14728222.2013.818656 (2013).

37 Sanders, O. \& Rajagopal, L. Phosphodiesterase Inhibitors for Alzheimer's Disease: A Systematic Review of Clinical Trials and Epidemiology with a Mechanistic Rationale. J Alzheimers Dis Rep 4, 185-215, doi:10.3233/ADR-200191 (2020).

38 Tibbo, A. J. \& Baillie, G. S. Phosphodiesterase 4B: Master Regulator of Brain Signaling. Cells $\mathbf{9}$, 1254 (2020). 
Basmarke-Wehelie, R. et al. The complement regulator CD46 is bactericidal to Helicobacter pylori and blocks urease activity. Gastroenterology 141, 918-928, doi:10.1053/j.gastro.2011.05.009 (2011). Nho, K. et al. Genome-wide transcriptome analysis identifies novel dysregulated genes implicated in Alzheimer's pathology. Alzheimer's \& Dementia 16, 1213-1223 (2020). Bagchi, S., Genardi, S. \& Wang, C.-R. Linking CD1-Restricted T Cells With Autoimmunity and Dyslipidemia: Lipid Levels Matter. Frontiers in Immunology 9, doi:10.3389/fimmu.2018.01616 (2018).

42 Chen, H. et al. Association between serum cholesterol levels and Alzheimer's disease in China: a case-control study. International Journal of Food Sciences and Nutrition 70, 405-411, doi:10.1080/09637486.2018.1508426 (2019).

43 Kao, Y.-C., Ho, P.-C., Tu, Y.-K., Jou, I.-M. \& Tsai, K.-J. Lipids and Alzheimer's Disease. International Journal of Molecular Sciences 21, 1505 (2020).

44 Kao, Y.-C., Ho, P.-C., Tu, Y.-K., Jou, I. \& Tsai, K.-J. Lipids and Alzheimer's disease. Int J Mol Sci 21, 1505 (2020).

45 Lamb, A. \& Chen, L.-F. Role of the Helicobacter pylori-induced inflammatory response in the development of gastric cancer. J Cell Biochem 114, 491-497, doi:10.1002/jcb.24389 (2013).

46 Iwai, N. et al. Helicobacter pylori eradication increases the serum high-density lipoprotein cholesterol level in the infected patients with chronic gastritis: A single-center observational study. PLOS ONE 14, e0221349, doi:10.1371/journal.pone.0221349 (2019).

47 Di Paolo, G. \& Kim, T.-W. Linking lipids to Alzheimer's disease: cholesterol and beyond. Nature Reviews Neuroscience 12, 284-296 (2011).

48 Kojro, E., Gimpl, G., Lammich, S., März, W. \& Fahrenholz, F. Low cholesterol stimulates the nonamyloidogenic pathway by its effect on the $\alpha$-secretase ADAM 10. Proceedings of the national academy of sciences 98, 5815-5820 (2001).

49 Vance, J. E., Hayashi, H. \& Karten, B. Cholesterol homeostasis in neurons and glial cells. Seminars in Cell \& Developmental Biology 16, 193-212, doi:https://doi.org/10.1016/j.semcdb.2005.01.005 (2005).

50 Henness, S. \& Perry, C. M. Orlistat. Drugs 66, 1625-1656, doi:10.2165/00003495-20066612000012 (2006).

51 Du, J. \& Wang, Z. Therapeutic potential of lipase inhibitor orlistat in Alzheimer's disease. Medical Hypotheses 73, 662-663, doi:https://doi.org/10.1016/j.mehy.2009.04.046 (2009).

52 Lin, C.-J. et al. Statin Therapy Is Associated with Reduced Risk of Peptic Ulcer Disease in the Taiwanese Population. Front Pharmacol 8, 210-210, doi:10.3389/fphar.2017.00210 (2017).

53 Pantea, M. et al. Statins and gastroduodenal endoscopic lesions: A case-control study. Medicine (Baltimore) 97, e13579-e13579, doi:10.1097/MD.0000000000013579 (2018).

54 Nseir, W. et al. Randomised clinical trial: simvastatin as adjuvant therapy improves significantly the Helicobacter pylori eradication rate--a placebo-controlled study. Aliment Pharmacol Ther 36, 231-238, doi:10.1111/j.1365-2036.2012.05161.x (2012).

55 Schultz, B. G., Patten, D. K. \& Berlau, D. J. The role of statins in both cognitive impairment and protection against dementia: a tale of two mechanisms. Transl Neurodegener 7, 5-5, doi:10.1186/s40035-018-0110-3 (2018).

56 Torrandell-Haro, G. et al. Statin therapy and risk of Alzheimer's and age-related neurodegenerative diseases. Alzheimer's \& Dementia: Translational Research \& Clinical Interventions 6, e12108, doi:https://doi.org/10.1002/trc2.12108 (2020).

57 van den Brink, A. C., Brouwer-Brolsma, E. M., Berendsen, A. A. M. \& van de Rest, O. The Mediterranean, Dietary Approaches to Stop Hypertension (DASH), and Mediterranean-DASH Intervention for Neurodegenerative Delay (MIND) Diets Are Associated with Less Cognitive Decline and a Lower Risk of Alzheimer's Disease-A Review. Advances in Nutrition 10, 10401065, doi:10.1093/advances/nmz054 (2019). 
58 Elmaliklis, I.-N. et al. Increased Functional Foods' Consumption and Mediterranean Diet Adherence May Have a Protective Effect in the Appearance of Gastrointestinal Diseases: A Case-Control Study. Medicines (Basel) 6, 50, doi:10.3390/medicines6020050 (2019).

59 Purcell, S. et al. PLINK: a tool set for whole-genome association and population-based linkage analyses. The American journal of human genetics 81, 559-575 (2007).

60 Bulik-Sullivan, B. K. et al. LD Score regression distinguishes confounding from polygenicity in genome-wide association studies. Nature Genetics 47, 291-295, doi:10.1038/ng.3211 (2015).

61 Han, B. \& Eskin, E. Random-effects model aimed at discovering associations in meta-analysis of genome-wide association studies. The American Journal of Human Genetics 88, 586-598 (2011).

62 Lee, C. H., Eskin, E. \& Han, B. Increasing the power of meta-analysis of genome-wide association studies to detect heterogeneous effects. Bioinformatics 33, i379-i388, doi:10.1093/bioinformatics/btx242 (2017).

63 Watanabe, K., Taskesen, E., van Bochoven, A. \& Posthuma, D. Functional mapping and annotation of genetic associations with FUMA. Nature Communications 8, 1826, doi:10.1038/s41467-017-01261-5 (2017).

64 Adewuyi, E. O. et al. Shared Molecular Genetic Mechanisms Underlie Endometriosis and Migraine Comorbidity. Genes 11, 268 (2020).

65 Sekula, P., Del Greco M, F., Pattaro, C. \& Köttgen, A. Mendelian Randomization as an Approach to Assess Causality Using Observational Data. J Am Soc Nephrol 27, 3253-3265, doi:10.1681/ASN.2016010098 (2016).

66 Bowden, J., Davey Smith, G., Haycock, P. C. \& Burgess, S. Consistent estimation in Mendelian randomization with some invalid instruments using a weighted median estimator. Genetic epidemiology 40, 304-314 (2016).

67 Bowden, J., Davey Smith, G. \& Burgess, S. Mendelian randomization with invalid instruments: effect estimation and bias detection through Egger regression. International journal of epidemiology 44, 512-525 (2015).

68 Verbanck, M., Chen, C.-y., Neale, B. \& Do, R. Detection of widespread horizontal pleiotropy in causal relationships inferred from Mendelian randomization between complex traits and diseases. Nature genetics 50, 693-698 (2018).

69 Cuéllar-Partida, G. et al. Complex-Traits Genetics Virtual Lab: A community-driven web platform for post-GWAS analyses. bioRxiv, 518027, doi:10.1101/518027 (2019).

70 Reimand, J. et al. Pathway enrichment analysis and visualization of omics data using g: Profiler, GSEA, Cytoscape and EnrichmentMap. Nature Protocols 14, 482-517, doi:10.1038/s41596018-0103-9 (2019).

\section{Author contributions}

Conceived the Study: EOA., TP., SML; designed the study: EOA., EKO., TP., SML.; conducted the analysis: EOA; interpreted the results: EOA., EKO., DRN., TP., SML.; drafted the manuscript: EOA.; made critical revisions to the manuscript: EOA., EKO., DRN., TP., SML; Funding: SML

\section{Competing interests}

All authors declare no competing interests 


\section{Genetic overlap between AD and GIT disorders Tables and Figures}

Table 1: Summary of GWAS datasets analysed

851

\begin{tabular}{|c|c|c|c|c|c|}
\hline $\mathbf{S} / \mathbf{N}$ & GWAS & Cases & Control & Sample size & Ancestry \\
\hline 1 & Clinically diagnosed AD and AD-by-proxy & 71,880 & 383,378 & 455,258 & European \\
\hline 2 & Gastroesophageal Reflux Disease (GERD) & 71,522 & 261,079 & 332,601 & \\
\hline 3 & Gastritis-Duodenitis-UKBB_SAIGE_PheCode_535 & 28,941 & 378,124 & 407,065 & \\
\hline 4 & Gastritis-Duodenitis & 14,477 & 286,314 & 300,791 & \\
\hline 5 & Peptic ulcer disease (PUD) & 16,666 & 439,661 & 456,327 & \\
\hline 6 & Gastroosophageal Reflux Disease (GORD) & 54,854 & 401,473 & 456,327 & \\
\hline 7 & Irritable Bowel Syndrome (IBS) & 28,518 & 426,803 & 455,321 & \\
\hline 8 & Inflammatory Bowel Disease (IBD) & 7045 & 426803 & 456,327 & \\
\hline 9 & PUD and GERD medication & 90,175 & 366,152 & 456,327 & \\
\hline 10 & Diverticulosis and diverticulitis-UKBB_PheCode_562 & 27,311 & 334,783 & 362,094 & \\
\hline 11 & Diverticular Disease & 14,028 & 286,763 & 300,791 & \\
\hline 12 & Lansoprazole & 13,559 & 266,884 & 280,443 & \\
\hline 13 & IBD (from GWAS Atlas) & 12,882 & 21,770 & 34,652 & \\
\hline
\end{tabular}

AD: Alzheimer's disease, GERD: gastroesophageal reflux disease, PUD: peptic ulcer disease, GORD: gastroesophageal reflux disease from another source, IBS: irritable bowel disease, IBD: inflammatory bowel disease. Note: the GWAS were obtained from multiple sources as more comprehensively described in Supplementary Table 1. 
Table 2: Results of genetic overlap assessment between AD (P1) and GERD (P2)

877

\begin{tabular}{lllllll}
\hline $\mathbf{P 1}$ & $\mathbf{P 2}$ & $\begin{array}{l}\text { Ind-SNPs- } \\
\text { overlapping }\end{array}$ & Concordant & $\begin{array}{l}\text { Prop- } \\
\text { Overlap }\end{array}$ & OR & $\begin{array}{l}\text { P } \\
\text { Fishers-exact }\end{array}$ \\
\hline $\mathbf{1}$ & $\leq 1$ & 26963 & 13955 & 0.52 & 1.18 & $4.65 \times 10^{-11}$ \\
\hline $\mathbf{0 . 9}$ & 0.9 & 24237 & 12653 & 0.52 & 1.19 & $7.46 \times 10^{-12}$ \\
\hline $\mathbf{0 . 8}$ & 0.8 & 21551 & 11289 & 0.52 & 1.21 & $3.02 \times 10^{-12}$ \\
\hline $\mathbf{0 . 7}$ & 0.7 & 18759 & 9860 & 0.53 & 1.23 & $2.64 \times 10^{-12}$ \\
\hline $\mathbf{0 . 6}$ & 0.6 & 16001 & 8483 & 0.53 & 1.27 & $2.48 \times 10^{-14}$ \\
\hline $\mathbf{0 . 5}$ & 0.5 & 13297 & 7081 & 0.53 & 1.30 & $7.54 \times 10^{-14}$ \\
\hline $\mathbf{0 . 4}$ & 0.4 & 10432 & 5603 & 0.54 & 1.35 & $3.42 \times 10^{-14}$ \\
\hline $\mathbf{0 . 3}$ & 0.3 & 7638 & 4173 & 0.55 & 1.45 & $6.22 \times 10^{-16}$ \\
\hline $\mathbf{0 . 2}$ & 0.2 & 4936 & 2755 & 0.56 & 1.6 & $2.86 \times 10^{-16}$ \\
\hline $\mathbf{0 . 1}$ & 0.1 & 2283 & 1315 & 0.58 & 1.85 & $4.06 \times 10^{-13}$ \\
\hline $\mathbf{0 . 0 5}$ & 0.05 & 1015 & 585 & 0.58 & 1.84 & $1.74 \times 10^{-6}$ \\
\hline $\mathbf{0 . 0 5}$ & 0.01 & 322 & 199 & 0.62 & 2.59 & $3.66 \times 10^{-5}$ \\
\hline $\mathbf{0 . 0 1}$ & 0.05 & 439 & 152 & 0.61 & 2.39 & $1.03 \times 10^{-5}$ \\
\hline $\mathbf{0 . 0 1}$ & 0.01 & 0.61 & 2.34 & $1.47 \times 10^{-2}$ \\
\hline
\end{tabular}

AD: Alzheimer's disease, GERD: gastroesophageal reflux disease, $P 1: P$-value for the dataset, $P 2$ : $P$-value for dataset 2, P1P2snp: Independent SNPs overlapping AD (P1) and GERD (P2) at each of the SNP subsets, Concord: number of concordant SNPs, SNP: Single Nucleotide Polymorphism, Ftest: Fisher's Exact test, OR: Odds ratio for the effect direction concordance association test for P1 and P2. Pval: Fisher's exact P-value for the effect direction concordance association test between AD (P1) and GERD (P2). 
Table 3: Genetic correlation between AD and GIT disorders

\begin{tabular}{|c|c|c|c|c|c|c|}
\hline Phenotype 1 & Phenotype 2 & $r_{g}$ & se & $\mathbf{P}$ & Gencov_int & gencov se \\
\hline \multirow{8}{*}{$\begin{array}{l}\text { AD without APOE } \\
\text { and } M H C \text { regions }\end{array}$} & $A D$ & 1.00 & $1.97 \times 10^{-6}$ & 0.00000 & 0.995 & 0.008 \\
\hline & GERD & 0.19 & $3.82 \times 10^{-2}$ & $8.78 \times 10^{-7}$ & 0.015 & 0.005 \\
\hline & Gastritis-and-duodenitis & 0.19 & $6.68 \times 10^{-2}$ & $5.40 \times 10^{-3}$ & 0.008 & 0.005 \\
\hline & PGM & 0.15 & $3.99 \times 10^{-2}$ & $1.43 \times 10^{-4}$ & 0.016 & 0.006 \\
\hline & PUD & 0.26 & $7.10 \times 10^{-2}$ & $2.92 \times 10^{-4}$ & 0.004 & 0.005 \\
\hline & IBS & 0.16 & $6.91 \times 10^{-2}$ & $2.36 \times 10^{-2}$ & 0.004 & 0.005 \\
\hline & Diverticular disease & 0.18 & $5.78 \times 10^{-2}$ & $1.59 \times 10^{-3}$ & -0.004 & 0.006 \\
\hline & IBD & -0.05 & $5.90 \times 10^{-2}$ & $3.80 \times 10^{-1}$ & 0.010 & 0.006 \\
\hline Phenotype 1 & Phenotype 2 & $r_{g}$ & se & $\mathbf{P}$ & Gencov_int & gencov se \\
\hline \multirow[t]{2}{*}{ (B) } & $A D$ & 1.00 & $3.06 \times 10^{-6}$ & 0.00000 & 1.049 & 0.051 \\
\hline & GERD & 0.24 & $6.59 \times 10^{-2}$ & $3.00 \times 10^{-3}$ & 0.013 & 0.005 \\
\hline \multirow{7}{*}{$\begin{array}{l}\text { AD with APOE } \\
\text { region }\end{array}$} & Gastritis-and-duodenitis & 0.26 & $9.90 \times 10^{-1}$ & $8.60 \times 10^{-3}$ & 0.005 & 0.005 \\
\hline & PGM & 0.21 & $7.58 \times 10^{-2}$ & $6.30 \times 10^{-3}$ & 0.014 & 0.006 \\
\hline & PUD & 0.33 & $1.24 \times 10^{-1}$ & $7.50 \times 10^{-3}$ & 0.004 & 0.005 \\
\hline & IBS & 0.19 & $9.45 \times 10^{-2}$ & $4.44 \times 10^{-2}$ & 0.005 & 0.005 \\
\hline & Diverticular disease & 0.23 & $8.27 \times 10^{-2}$ & $4.66 \times 10^{-3}$ & -0.005 & 0.005 \\
\hline & Lansoprazole medication & 0.25 & $1.22 \times 10^{-1}$ & $3.72 \times 10^{-2}$ & 0.006 & 0.005 \\
\hline & IBD & -0.07 & $7.21 \times 10^{-2}$ & $3.27 \times 10^{-1}$ & 0.012 & 0.007 \\
\hline
\end{tabular}

AD: Alzheimer's disease, GIT: gastro-intestinal tract, GERD: gastroesophageal reflux disease, PUD: peptic ulcer disease, PGM: medications for GERD and PUD, IBS: irritable bowel syndrome, IBD: Inflammatory bowel disease, $r_{g}$ : genetic correlation, se: standard error, P: P-value, MHC: major histocompatibility complex, gencov_int: genetic covariance intercept. 
Table 4: Genome-wide significant independent SNPs and loci for AD and GIT disorders

\begin{tabular}{|c|c|c|c|c|c|c|c|c|c|c|c|c|c|c|}
\hline \multirow{2}{*}{$\begin{array}{l}\text { Independent } \\
\text { SNPs }\end{array}$} & \multirow[t]{2}{*}{ Locus } & \multirow[t]{2}{*}{ Lead SNPs } & \multirow[t]{2}{*}{ Chr } & \multirow[t]{2}{*}{ BP } & \multirow[t]{2}{*}{ EA } & \multirow[t]{2}{*}{ NEA } & \multirow[t]{2}{*}{$1^{2}$} & \multirow{2}{*}{$\begin{array}{l}\text { Nearest coding } \\
\text { genes/cytoband }\end{array}$} & \multicolumn{2}{|c|}{ Meta-analysis } & \multicolumn{2}{|l|}{$A D$} & \multicolumn{2}{|c|}{ GIT Disorders } \\
\hline & & & & & & & & & P-value & $\mathrm{BE}$ & P-value & M-value & P-value & M-value \\
\hline \multicolumn{15}{|c|}{ SNPs and loci reaching genome-wide significance after a meta-analysis of AD GWAS and PGM GWAS } \\
\hline rs2840677 & 1 & rs12058296 & 1 & 66333877 & A & $\mathrm{T}$ & 66.10 & PDE4B/ 1p31.3 & $2.43 \times 10^{-8}$ & $4.56 \times 10^{-8}$ & $5.73 \times 10^{-3}$ & 0.94 & $2.20 \times 10^{-7}$ & 1.00 \\
\hline rs6695557 & & & 1 & 66349013 & A & G & 57.16 & PDE4B/ 1p31.3 & $8.46 \times 10^{-9}$ & $2.08 \times 10^{-8}$ & $3.02 \times 10^{-3}$ & 0.97 & $1.89 \times 10^{-7}$ & 1.00 \\
\hline rs12058296 & & & 1 & 66402424 & A & C & 84.60 & PDE4B/ 1p31.3 & $5.02 \times 10^{-9}$ & $9.37 \times 10^{-8}$ & $3.22 \times 10^{-5}$ & 0.99 & $8.68 \times 10^{-6}$ & 1.00 \\
\hline rs4147104 & 2 & rs4147104 & 1 & 207882194 & A & G & 13.54 & CD46 / 1q32.2 & $5.47 \times 10^{-9}$ & $1.48 \times 10^{-8}$ & $1.02 \times 10^{-6}$ & 1.00 & $6.12 \times 10^{-4}$ & 1.00 \\
\hline rs709210 & 3 & rs7642934 & 3 & 50357869 & A & C & 93.99 & HYAL2 / 3p21.31 & $4.39 \times 10^{-8}$ & $1.59 \times 10^{-7}$ & $1.55 \times 10^{-2}$ & NA & $6.28 \times 10^{-8}$ & NA \\
\hline rs7642934 & & & 3 & 50174848 & A & G & 93.64 & SEMA3F / 3p21.31 & $2.78 \times 10^{-8}$ & $1.51 \times 10^{-7}$ & $7.47 \times 10^{-3}$ & 0.01 & $8.19 \times 10^{-8}$ & 1.00 \\
\hline rs2858331 & 4 & rs2858331 & 6 & 32681277 & G & A & 61.35 & HLA-DQA2/ 6p21.32 & $3.08 \times 10^{-10}$ & $7.14 \times 10^{-10}$ & $1.24 \times 10^{-7}$ & 1.00 & $1.18 \times 10^{-4}$ & 1.00 \\
\hline rs28895026 & & & 6 & 32391695 & C & $\mathrm{T}$ & 0.00 & HLA-DRA/6p21.32 & $5.43 \times 10^{-9}$ & $1.38 \times 10^{-8}$ & $1.48 \times 10^{-7}$ & 1.00 & $8.38 \times 10^{-3}$ & 0.98 \\
\hline rs34644948 & 5 & rs34644948 & 16 & 70681658 & $\mathrm{~T}$ & C & 0.00 & MTSS2 / 16q22.1 & $2.11 \times 10^{-8}$ & $5.63 \times 10^{-8}$ & $1.98 \times 10^{-7}$ & 1.00 & $3.13 \times 10^{-2}$ & 0.95 \\
\hline rs2584662 & 6 & rs2584662 & 17 & 47470487 & C & $A$ & 0.00 & PHB / 17q21.33 & $3.94 \times 10^{-9}$ & $1.00 \times 10^{-8}$ & $1.98 \times 10^{-7}$ & 1.00 & $4.91 \times 10^{-3}$ & 0.99 \\
\hline rs11083749 & 7 & rs11083749 & 19 & 45384105 & $T$ & C & 0.00 & APOE / 19q13.32 & $2.84 \times 10^{-8}$ & $7.35 \times 10^{-8}$ & $1.98 \times 10^{-7}$ & 1.00 & $3.45 \times 10^{-2}$ & 0.95 \\
\hline \multicolumn{15}{|c|}{ SNPs and loci reaching genome-wide significance after a meta-analysis of AD GWAS and GERD GWAS } \\
\hline rs12058296 & 1 & rs12058296 & 1 & 66402424 & A & C & 85.75 & PDE4B/ 1p31.3 & $1.05 \times 10^{-8}$ & $2.10 \times 10^{-7}$ & $3.22 \times 10^{-5}$ & 0.99 & $1.74 \times 10^{-5}$ & 1.00 \\
\hline rs2503185 & & & 1 & 66461401 & G & A & 91.93 & PDE4B/ 1p31.3 & $3.44 \times 10^{-8}$ & $1.21 \times 10^{-8}$ & $9.53 \times 10^{-4}$ & 0.25 & $9.60 \times 10^{-7}$ & 1.00 \\
\hline rs12561863 & 2 & rs12561863 & 1 & 190897608 & A & $\mathrm{T}$ & 96.02 & BRINP3/ 1q31.1 & $1.68 \times 10^{-8}$ & $2.89 \times 10^{-7}$ & $5.76 \times 10^{-3}$ & 0.00 & $1.05 \times 10^{-7}$ & 1.00 \\
\hline rs3774745* & 3 & rs3774745 & 3 & 50204745 & $T$ & C & 92.74 & SEMA3F/ 3p21.31* & $2.01 \times 10^{-9}$ & $2.57 \times 10^{-9}$ & $2.55 \times 10^{-4}$ & 0.36 & $1.64 \times 10^{-7}$ & 1.00 \\
\hline rs28895026 & 4 & rs28895026 & 6 & 32391695 & C & $\mathrm{T}$ & 0.00 & HLA-DRA/6p21.32 & $2.06 \times 10^{-8}$ & $5.37 \times 10^{-8}$ & $1.48 \times 10^{-7}$ & 1.00 & $4.26 \times 10^{-2}$ & 0.94 \\
\hline rs8067459 & 5 & rs2584662 & 17 & 47444113 & C & $\mathrm{T}$ & 0.00 & ZNF652/ 17q21.32 & $3.07 \times 10^{-8}$ & $8.09 \times 10^{-8}$ & $2.15 \times 10^{-7}$ & 1.00 & $4.48 \times 10^{-2}$ & 0.93 \\
\hline rs2584662 & & & 17 & 47470487 & C & $A$ & 0.00 & PHB/17q21.33 & $7.72 \times 10^{-9}$ & $1.96 \times 10^{-8}$ & $1.54 \times 10^{-7}$ & 1.00 & $1.02 \times 10^{-2}$ & 0.98 \\
\hline rs11083749 & 6 & rs1132899 & 19 & 45384105 & $T$ & C & 0.00 & APOE/ 19q13.32 & $2.63 \times 10^{-8}$ & $6.88 \times 10^{-8}$ & $2.46 \times 10^{-7}$ & 1.00 & $3.14 \times 10^{-2}$ & 0.95 \\
\hline rs1132899 & & & 19 & 45448036 & $T$ & C & 94.99 & APOC2/ 19q13.32 & $1.19 \times 10^{-8}$ & $1.57 \times 10^{-7}$ & $5.41 \times 10^{-8}$ & 1.00 & $5.53 \times 10^{-3}$ & 0.00 \\
\hline rs117501883 & 7 & rs117501883 & 19 & 45841296 & A & G & 0.00 & KLC3/ 19q13.32 & $8.96 \times 10^{-9}$ & $2.32 \times 10^{-8}$ & $7.13 \times 10^{-8}$ & 1.00 & $3.78 \times 10^{-2}$ & 0.94 \\
\hline rs76692930 & & & 19 & 45875851 & $T$ & C & 50.22 & ERCC2/ 19q13.32 & $3.51 \times 10^{-8}$ & $8.60 \times 10^{-8}$ & $3.18 \times 10^{-6}$ & 1.00 & $8.32 \times 10^{-4}$ & 0.99 \\
\hline
\end{tabular}


SNPs and loci reaching genome-wide significance after a meta-analysis of AD GWAS and PUD GWAS

\begin{tabular}{|c|c|c|c|c|c|c|c|c|c|c|c|c|c|c|}
\hline rs36133610 & 1 & rs36133610 & 2 & 234067884 & A & G & 0.00 & INPP5D/ 2q37.1 & $1.24 \times 10^{-8}$ & $3.41 \times 10^{-8}$ & $5.85 \times 10^{-8}$ & 1.00 & $4.90 \times 10^{-2}$ & 0.92 \\
\hline rs9270599 & 2 & rs9270599 & 6 & 32561656 & G & A & 26.29 & HLA-DRA/6p21.32 & $9.12 \times 10^{-9}$ & $2.59 \times 10^{-8}$ & $5.60 \times 10^{-8}$ & 1.00 & $2.72 \times 10^{-2}$ & 0.92 \\
\hline rs530324 & 3 & rs530324 & 8 & 27491186 & C & G & 76.92 & SCARA3/ 8p21.1 & $2.27 \times 10^{-8}$ & $6.17 \times 10^{-8}$ & $3.32 \times 10^{-7}$ & 1.00 & $2.00 \times 10^{-3}$ & 0.96 \\
\hline rs73976310 & 4 & rs73976310 & 17 & 5014212 & A & G & 31.11 & USP6/ 17p13.2 & $1.20 \times 10^{-8}$ & $3.67 \times 10^{-8}$ & $7.04 \times 10^{-8}$ & 1.00 & $2.70 \times 10^{-2}$ & 0.90 \\
\hline rs28363848 & 5 & rs28363848 & 19 & 45257201 & $T$ & G & 41.06 & BCL3/ 19q13.32 & $1.04 \times 10^{-8}$ & $2.96 \times 10^{-8}$ & $5.63 \times 10^{-8}$ & 1.00 & $2.60 \times 10^{-2}$ & 0.91 \\
\hline rs3852865 & 6 & rs3852865 & 19 & 51714065 & A & G & 58.20 & CD33/ 19q13.41 & $1.81 \times 10^{-8}$ & $5.19 \times 10^{-8}$ & $1.63 \times 10^{-7}$ & 1.00 & $9.90 \times 10^{-3}$ & 0.94 \\
\hline rs7245846 & & & 19 & 51731176 & A & G & 5.22 & CD33/ 19q13.41 & $2.32 \times 10^{-8}$ & $6.71 \times 10^{-8}$ & $1.19 \times 10^{-7}$ & 1.00 & $4.00 \times 10^{-2}$ & 0.91 \\
\hline
\end{tabular}

SNP: Single nucleotide polymorphism; Chr: Chromosome; EA: Effect allele; NEA: Non-effect allele; 12: I-square for heterogeneity assessment; Se: Standard error; P: P-value; Meta-analysis model use was RE2C and RE2. RE2C: GWAS meta-analysis methods that account for sample overlap and heterogeneity. RE2: adjusts for heterogeneity. ${ }^{*}$ The m-value suggests that the SNP 


\section{Table 5: Summary of MR analysis results for AD and GIT disorders}

\begin{tabular}{|c|c|c|c|c|c|c|c|c|c|c|c|c|c|c|}
\hline \multirow{2}{*}{$\begin{array}{l}\text { Exposure } \\
\text { (nSNPs) }\end{array}$} & \multirow[t]{2}{*}{ Outcome } & \multicolumn{2}{|l|}{ IVW } & \multicolumn{2}{|c|}{ Weighted median } & \multicolumn{2}{|c|}{ MR-Egger } & \multicolumn{5}{|l|}{ MR-PRESSO } & \multicolumn{2}{|c|}{ MR-Egger Intercept } \\
\hline & & Beta & $\mathbf{P}$ & Beta & $\mathbf{P}$ & Beta & $\mathbf{P}$ & Global test P & Raw Beta & $\mathbf{P}$ & Corrected Beta & $\mathbf{P}$ & Intercept & $\mathbf{P}$ \\
\hline$A D(28)$ & GERD & -0.053 & 0.266 & 0.011 & 0.860 & -0.059 & 0.362 & 0.113 & -0.052 & 0.276 & - & - & 0.00034 & 0.879 \\
\hline GERD (24) & $A D$ & 0.014 & 0.351 & -0.002 & 0.920 & -0.053 & 0.597 & 0.435 & 0.0136 & 0.361 & - & - & 0.0025 & 0.502 \\
\hline$A D(28)$ & PGM & -0.061 & 0.112 & -0.016 & 0.769 & -0.045 & 0.391 & 0.231 & -0.061 & 0.123 & - & - & -0.001 & 0.631 \\
\hline PGM (17) & $A D$ & 0.023 & 0.322 & -0.005 & 0.837 & -0.148 & 0.199 & 0.017 & 0.023 & 0.337 & 0.009 & 0.661 & 0.007 & 0.133 \\
\hline$A D(28)$ & PUD & 0.036 & 0.651 & 0.144 & 0.211 & 0.071 & 0.504 & 0.113 & 0.036 & 0.60 & - & - & -0.002 & 0.612 \\
\hline PUD (8) & $A D$ & 0.021 & 0.238 & 0.025 & 0.122 & 0.055 & 0.658 & 0.0104 & 0.021 & 0.277 & 0.0331 & 0.053 & -0.00291 & 0.770 \\
\hline$A D(28)$ & Diverticular & -0.055 & 0.597 & -0.214 & 0.105 & -0.12 & 0.397 & 0.094 & -0.055 & 0.601 & - & - & 0.0034 & 0.483 \\
\hline Diverticular (16) & $A D$ & -0.001 & 0.883 & -0.001 & 0.905 & 0.007 & 0.811 & 0.316 & -0.001 & 0.884 & - & - & -0.00076 & 0.773 \\
\hline$A D(28)$ & IBS* & 0.043 & 0.623 & 0.142 & 0.123 & -0.016 & 0.888 & 0.0012 & 0.043 & 0.626 & 0.010 & 0.892 & 0.0032 & 0.438 \\
\hline$A D(28)$ & Gastritis-D* & -0.085 & 0.267 & -0.101 & 0.273 & -0.173 & 0.098 & 0.034 & -0.085 & 0.277 & - & - & 0.0046 & 0.196 \\
\hline$A D(28)$ & IBD & 0.254 & 0.104 & 0.365 & 0.094 & 0.277 & 0.231 & 0.327 & 0.254 & 0.115 & - & - & -0.00097 & 0.885 \\
\hline IBD (24) & $A D$ & -0.0005 & 0.895 & -0.003 & 0.526 & 0.004 & 0.607 & 0.316 & -0.0005 & 0.895 & - & - & 0.00073 & 0.497 \\
\hline
\end{tabular}

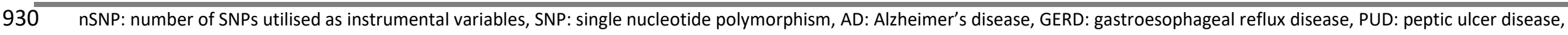

931 PGM: medications for GERD or PUD, Diverticular: diverticular disease, IBS: irritable bowel syndrome, IBD: inflammatory bowel disease, IVW: inverse variance weighted, P: P-value, MR-

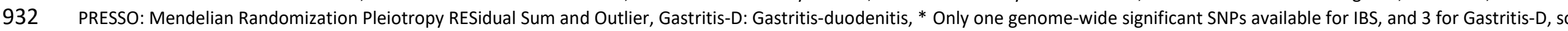

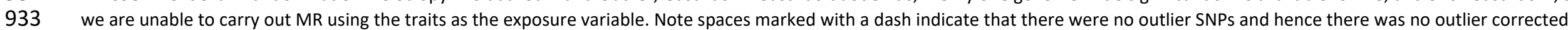

934 results in the MR-PRESSO analysis. 
Table 6: Partial causality assessment using the Latent Causal Variable approach

\begin{tabular}{lllllll}
\hline Trait 1 & Trait 2 & $\mathbf{G C P}$ & $\mathbf{S E}$ & $\mathbf{P}$ & $\mathbf{r}_{\mathbf{g}}$ & $\mathbf{r}_{\mathbf{g}}-\mathbf{P}$ \\
\hline AD & Gastritis-duodenitis (Main ICD10: K29) & $\mathbf{- 0 . 6 9}$ & $\mathbf{0 . 2 7}$ & $\mathbf{0 . 0 0 2 6}$ & 0.39 & 0.033 \\
\cline { 2 - 7 } & Gastritis-duodenitis (PheCode_535_SAIGE) & -0.36 & 0.39 & 0.43 & 0.25 & 0.018 \\
\cline { 2 - 7 } & PGM & -0.45 & 0.37 & 0.22 & 0.21 & 0.0064 \\
\hline & PUD & 0.49 & 0.32 & 0.24 & 0.33 & 0.0077 \\
\hline IBS & 0.35 & 0.29 & 0.38 & 0.19 & 0.043 \\
\hline GERD & -0.01 & 0.58 & 0.64 & 0.25 & 0.060 \\
\hline & Diverticular disease (Main ICD10: K57) & $\mathbf{0 . 2 3}$ & $\mathbf{0 . 1 0}$ & $\mathbf{0 . 0 0 0 2 7 2}$ & 0.26 & 0.0396 \\
\hline IBD & $\mathbf{- 0 . 5 2}$ & $\mathbf{0 . 4 4}$ & $\mathbf{0 . 0 0 2 3 1 9}$ & -0.0397 & 0.6832 \\
\hline
\end{tabular}

AD: Alzheimer's disease, GCP: Genetic causal proportion, SE: Standard error, P: P-value, $r_{g}$ : genetic correlation, rg-P: genetic correlation P-value, GERD: gastroesophageal reflux disease, PUD: peptic ulcer disease, PGM: GWAS for medication use in GERD and PUD, IBD: inflammatory bowel disease.

940

941 


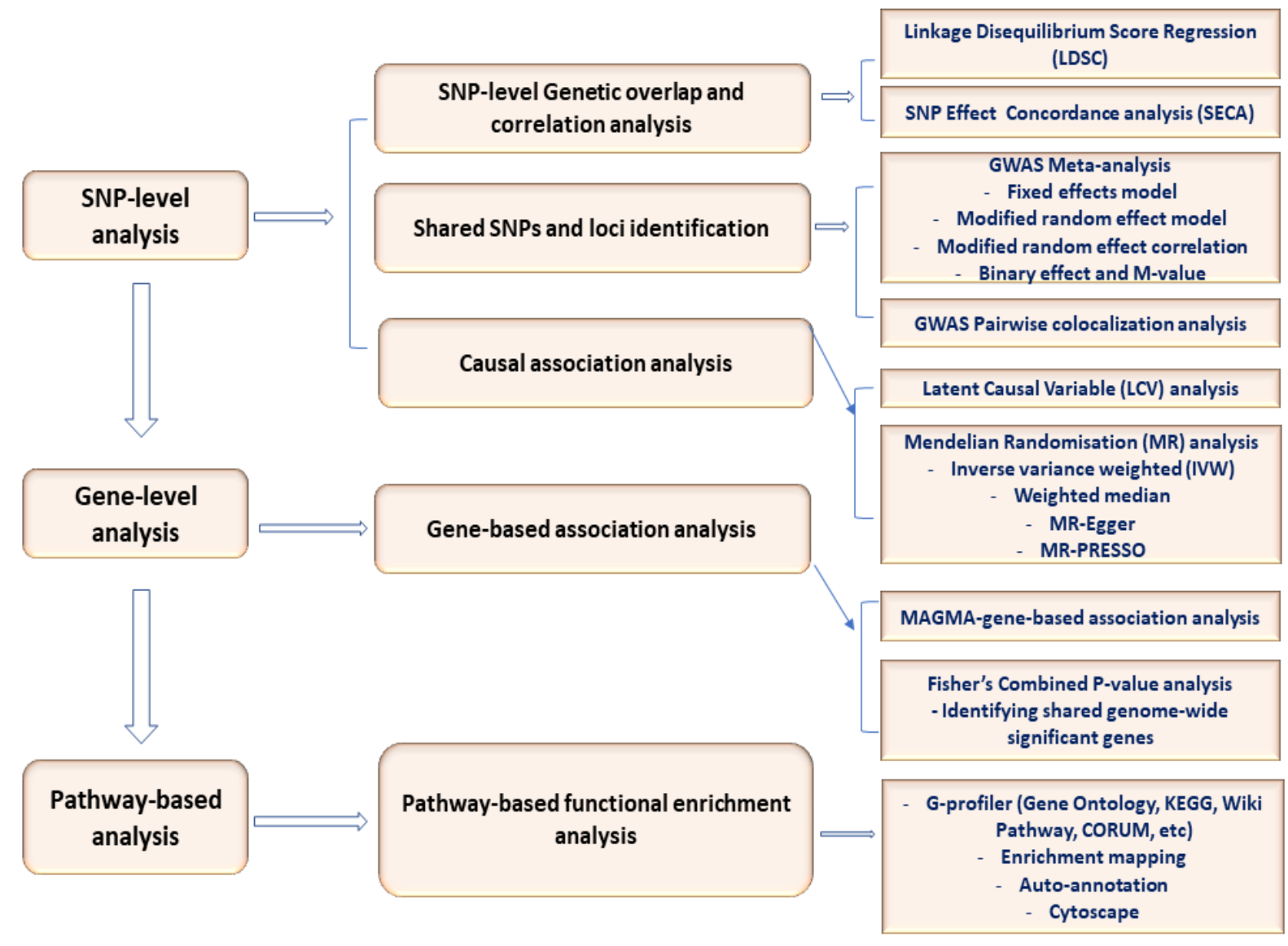

947 Fig 1: Simplified workflow for AD and GIT disorders relationship analysis 


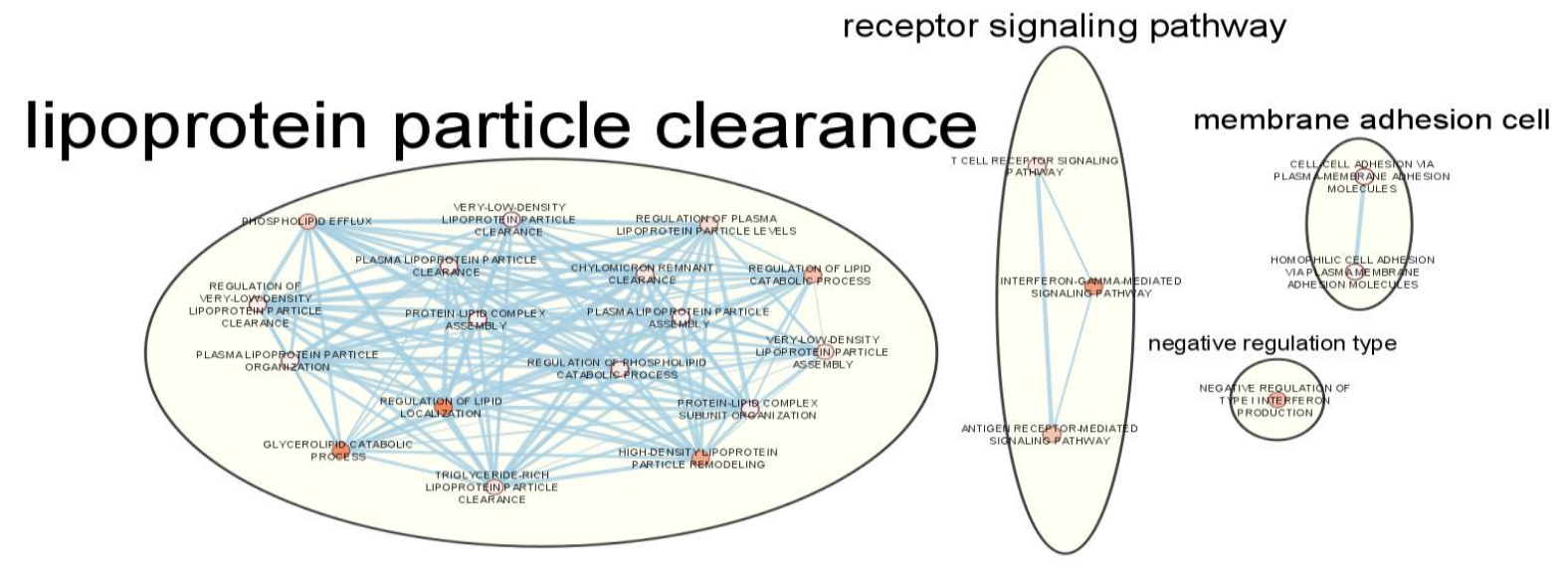

954

955

956

957

958

959

2. Gene Ontology: Cellular Components

\section{side membrane vesicle}

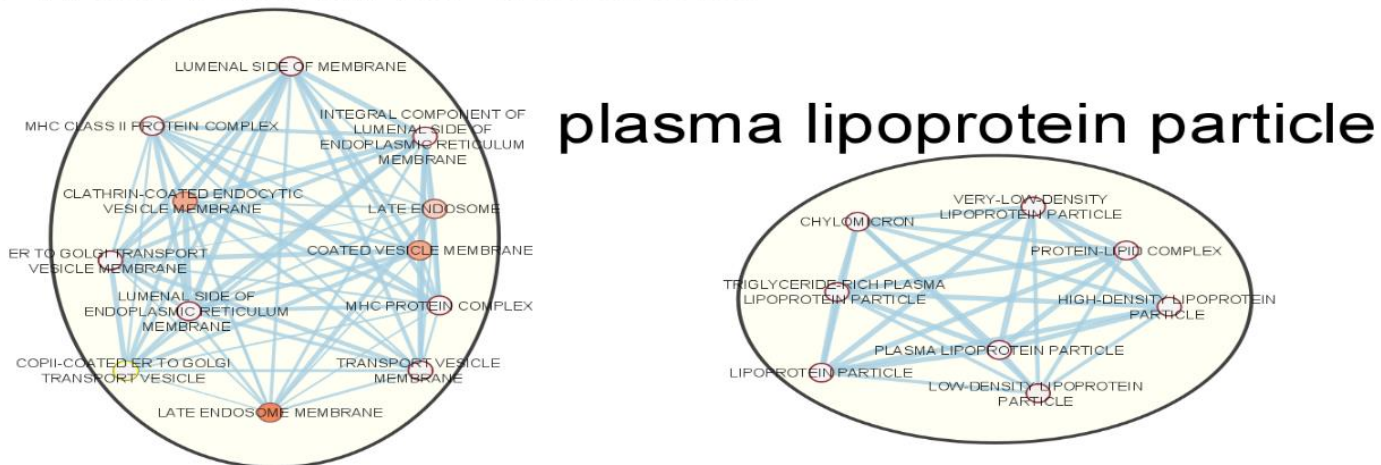

3. Gene Ontology: Molecular Function

\section{peptide antigen binding}

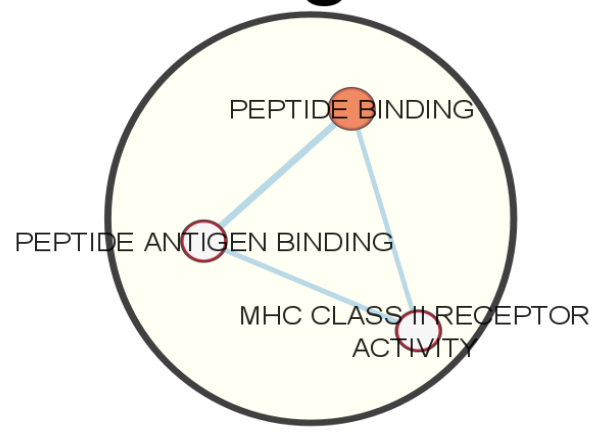

lipase inhibitor activity
4. KEGG: Kyoto Encyclopedia of Genes and Genomes

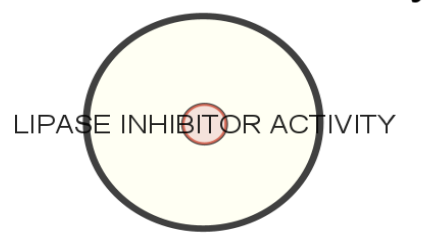




\section{intestinal immune network}

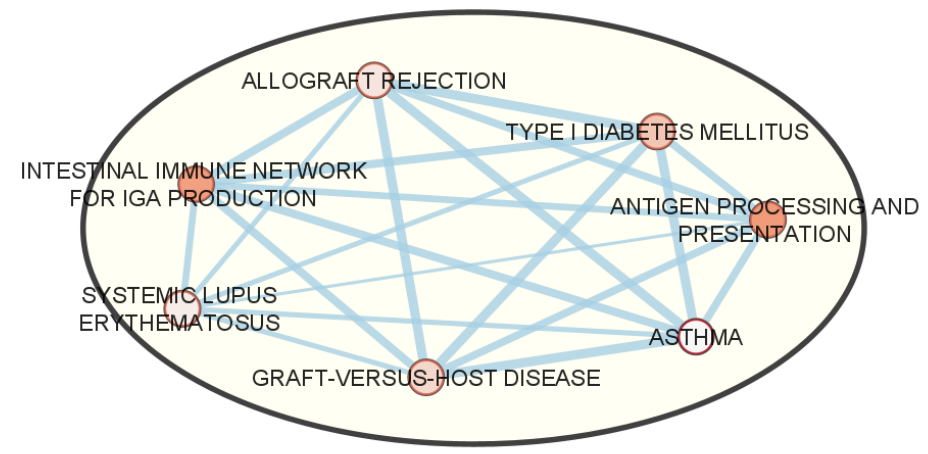

cholesterol metabolism

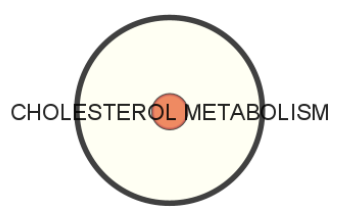

5. Reactome, Wiki pathway and Transcription Factor Binding sight

\section{assembly clearance plasma}

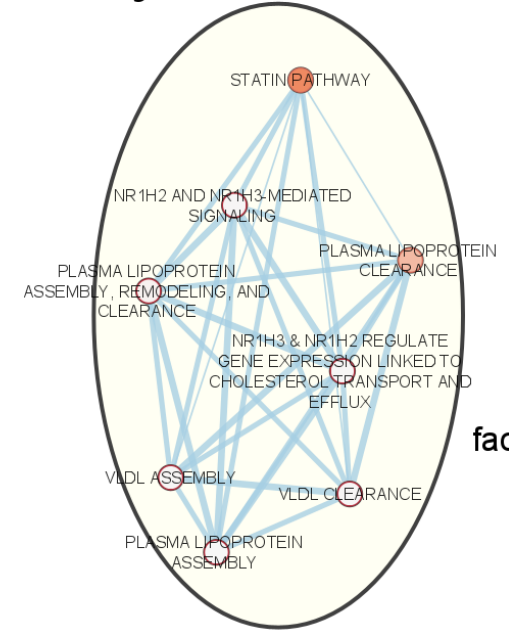

\section{interferon gamma signaling}

Figure 2: Clusters of significantly enriched biological pathways for AD and GERD 


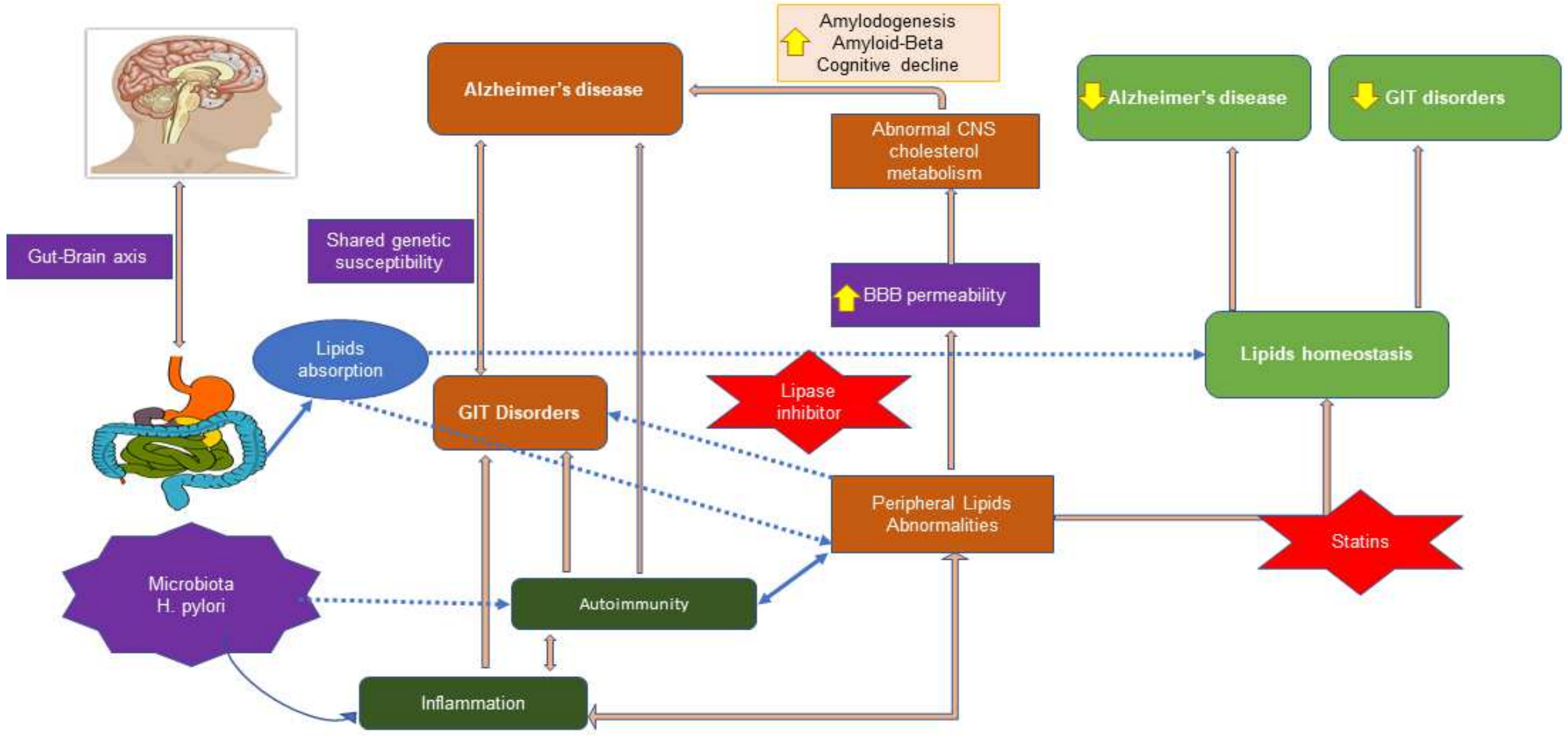

\section{Figure 3: Summary of findings suggesting potential mechanisms of $A D$ and GIT disorders}

GIT: gastrointestinal tract, H. pylori: Helicobacter pylori, CNS: central nervous system, BBB: the blood-brain barrier. Findings in this study indicate shared genetic susceptibility in $A D$ and GIT disorders, consistent with the concept of the gut-brain axis and the suggestions of some observational studies. Potential mechanisms implicated include those related to autoimmunity, lipids metabolism, lipase inhibitors and statins pathways. There is a connection between autoimmunity and dyslipidaemia, and consistent with our findings, previous studies suggest lipids play a role in both AD and GIT disorders. For example, hypercholesterolemia is believed to increase the permeability of BBB, facilitating the entry of peripheral cholesterol into the CNS, resulting in abnormal cholesterol metabolism in the brain, and subsequently in AD pathology. Similarly, H. pylori is believed to cause or worsen abnormal serum lipid profiles through chronic inflammatory processes. Lipid inhibitors and statins may thus find therapeutic applications in these disorders or their comorbidity. 


\section{Supplementary Files}

This is a list of supplementary files associated with this preprint. Click to download.

- SupplementaryNotesandfiguresADGITdisordermanuscript.pdf

- SupplementaryTableADGITdisordermanuscript.xIsx 